\title{
High-speed imaging in fluids
}

\section{Michel Versluis}

Version: November 22, 2012

\begin{abstract}
High-speed imaging is in popular demand for a broad range of experiments in fluids. It allows for a detailed visualization of the event under study by acquiring a series of image frames captured at high temporal and spatial resolution. This review covers high-speed imaging basics, by defining criteria for high-speed imaging experiments in fluids and to give rule-of-thumbs for a series of cases. It also considers stroboscopic imaging, triggering and illumination, and scaling issues. It provides guidelines for testing and calibration. Ultra high-speed imaging at frame rates exceeding 1 million frames per second is reviewed, and the combination of conventional experiments in fluids techniques with high-speed imaging techniques are discussed. The review is concluded with a high-speed imaging chart, which summarizes criteria for temporal scale and spatial scale and which facilitates the selection of a high-speed imaging system for the application.
\end{abstract}

Keywords flow visualization · ultra high-speed imaging

PACS 42.79.Pw $\cdot$ 42.65.Re

Michel Versluis

Physics of Fluids Group, MESA+ Institute of Nanotechnology, MIRA Institute of Biomedical Technology and Technical Medicine, University of Twente

P.O. Box 217, 7500 AE Enschede, The Netherlands

Tel.: +31 53489 8077, Fax: +31 534898068

E-mail: m.versluis@utwente.nl 


\section{Introduction}

The beauty of slow-motion movies captured with high-speed imaging has traditionally been described along the words 'making the invisible visible', 'seeing is believing', 'seeing the unseen', 'making flow motion into slow motion', 'science or art', or 'capturing the moment'. In recent years high-speed imaging has taken a groundbreaking step forward in our scientific world of flow research and improved high-speed imaging technology has pushed new and exciting insights in the physical mechanisms underlying flow and flow-related phenomena. Typical applications of high-speed imaging include car crash testing, air bag deployment, machine vision technology for packing and sorting, high-speed impact and materials testing, sport science, ballistics and (nuclear) detonation and explosions. In fluid dynamics applications of high-speed imaging include propulsion and cavitation, combustion, turbines and supersonic flows, sprays and jets, and shockwaves. Emerging applications in microfluidics, in biomedicine and biomechanics require top-of-the-line high-speed imaging systems, i.e. high frame rates at high spatial resolution at a high number of frames. Recent examples of these applications include animal locomotion, such as that of the water striders [1], of insect flight [2], of the Common Basilisk (Basiliscus basiliscus) or Jezus Christ lizard that runs on the surface of water [3], and that of the snapping shrimp [4,5] and Mantis shrimp [6]. In microfluidics we find applications in pinch-off phenomena in a dripping faucet $[7,8]$, splashing [9-11], in jet instabilities and jet break-up [12,13], and in ink jet printing [14,15]. In medicine, time-resolved dynamics of ultrasound contrast agent microbubbles insonified at $\mathrm{MHz}$ frequencies [16-19], shockwave lithotripsy for controlled kidney stone fracturing [20], and cell membrane permeabilization through acoustic streaming and sonoporation [21-24] are all to be visualized at nanoseconds timescale.

Probably the most striking icon in high-speed flow visualization is the crown-shaped splash made by a milk droplet, Fig. 1, as it hits the surface of a liquid captured by Harold Edgerton in 1936 using flash photography, Fig. 1B. Edgerton, who was the inventor of the high-speed strobe flash light, was and is a master in high-speed still photography. He produced pictures of hummingbirds in flight [26], a water jet from a faucet [27], and the vortical air movement near spinning fan blades [28] by freezing the motion of the object using his microseconds flash technique. Edgerton was not the first to discover the crown-shaped splash, as it was Worthington [29,30], who worked on splashes for all of his career in studies of impacting solids and fluids, and who made the first studies on the crown-shaped splash by carefully drawing out the contours of the impact phenomenon after a 3 microsecond spark plug illumination in 1877, see Fig 1A, after earlier work in high-speed photography using spark discharges by Fox Talbot [31], Cranz [32], and Ernst Mach [33]. The Worthington jet, which arises when a sphere 


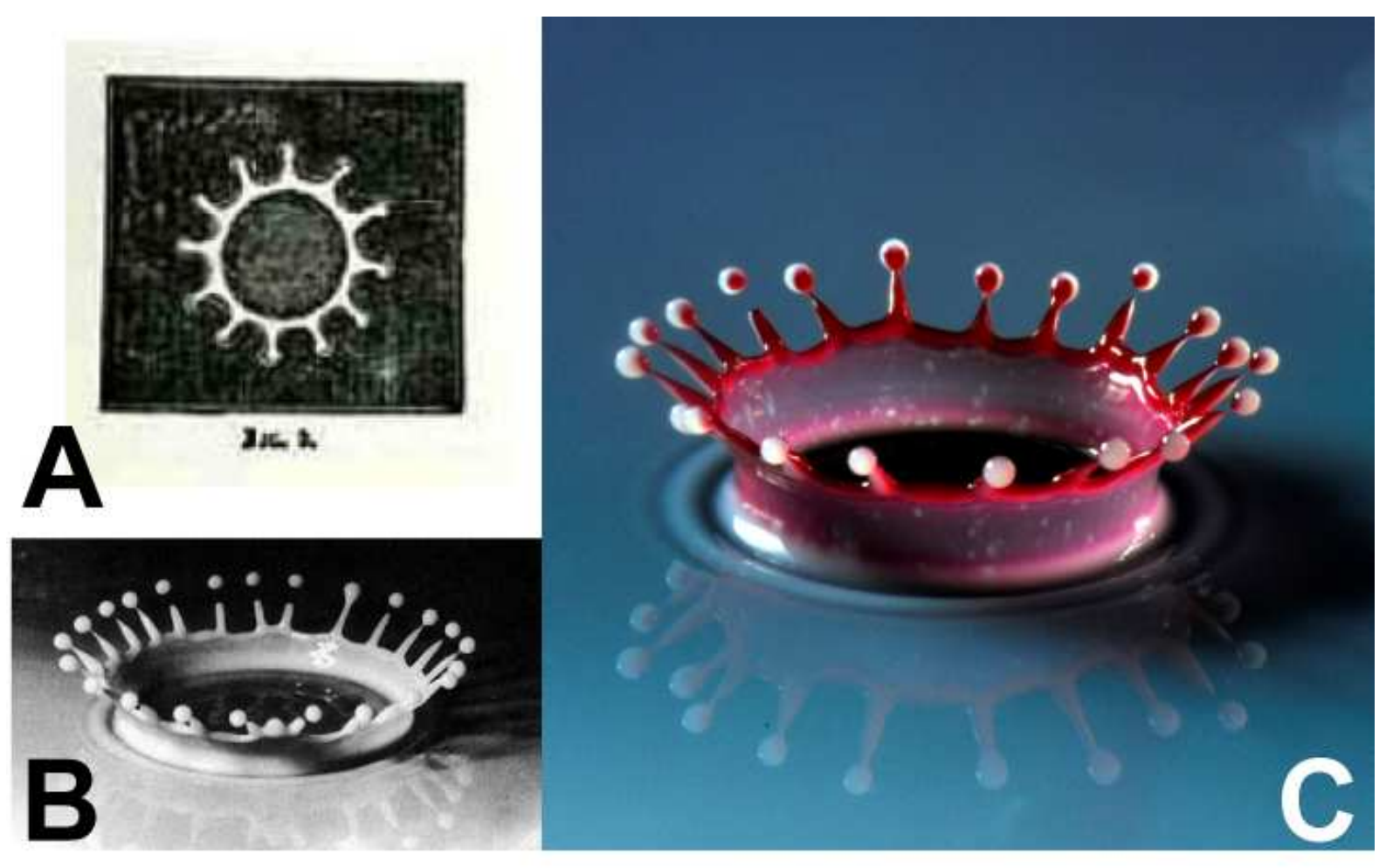

Fig. 1 A: A liquid splash drawn by Worthington using spark illumination. B: High-speed photography of the milk drop coronet by Harold Edgerton, the inventor of the electronic flash stroboscope (1936). The milk drop hits a thin layer of fluid, spreads and creates a corona which breaks up due to surface tension [25]. C: A Splash of Red: a 2-mm droplet of red dye impacting on a thin layer of milk. High-speed photography reveals crown formation with tips of entrained milk covering the rim of the coronet. Jets extend from the tips, breaking up the white satellite droplets with a splash of red (movie online).

or a droplet plunges into a pool of water, creating a cavity which collapses under the water pressure, forming an up-shooting jet, is named after him. His remarkably detailed piece of work (A Study of Splashes [34]) is an inspiring masterpiece which is still very attractive and actual as we speak. And indeed, the use of high-speed flash photography is still highly appropriate today. In fact, still photography is the preferred option for high-speed flow visualization. It is inexpensive, it is flexible, it has superior resolution and dynamic range and provided that the illumination pulse is short enough, it has excellent motion arrest. Flash photography should in fact come first to mind when studying high-speed flow phenomena [35]. We have investigated the milk coronet formed after the impact of a red-colored milk droplet as it hits the surface, see Fig. 1C. The image was taken with a 12.3 Mpixel Nikon D300 SLR digital photo camera connected to a programmable trigger/delay unit and a high-speed flash source. The photograph reveals that only the inside of the crown is coated with the fluid of the impacting droplet, which is most prominently visible at the level of the satellite droplets, formed after pinch-off of the crown jets, and which are half covered with a splash of red dye. Experimentally, the situation becomes much more complex when the flow phenomenon under study is dynamic. We can have high-speed events, transients and when the phenomenon is non-reproducible, non-repetitive or non-localized in time high-speed flash photography will be of 


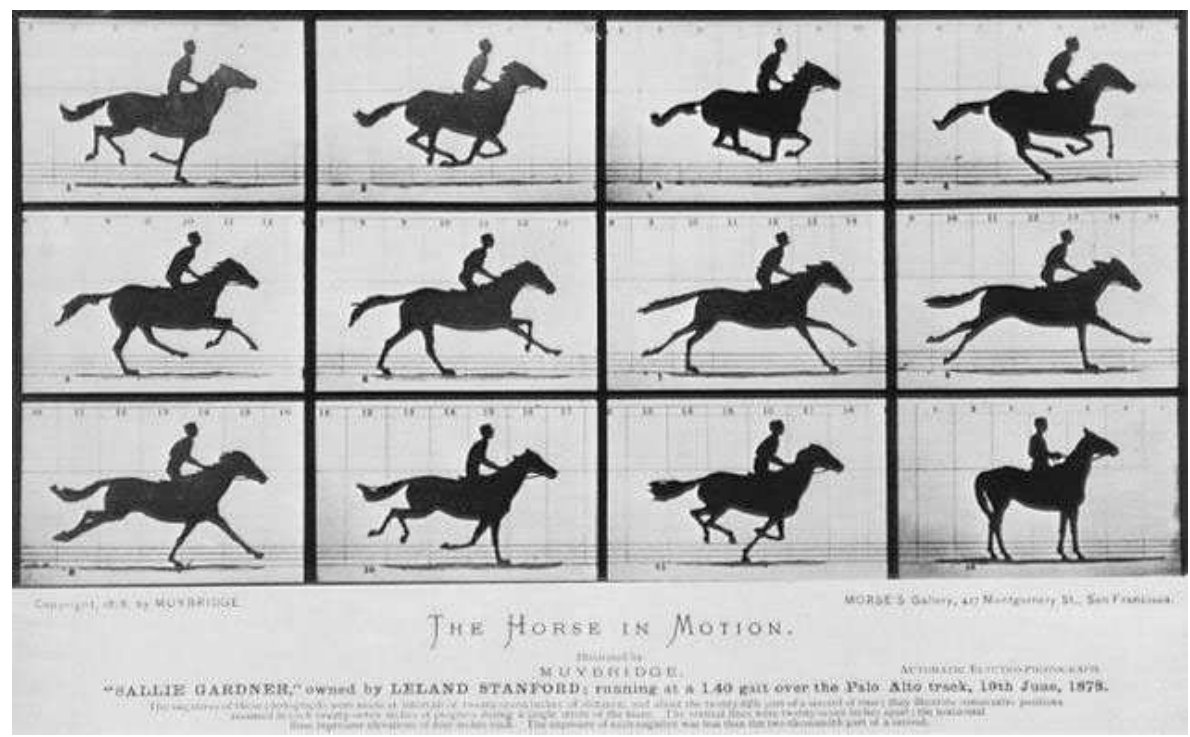

Fig. 2 The galloping horse taken by Eadweard Muybridge (1878). First high-speed imaging with 12 cameras at 1 ms interframe time. Settled the question whether during a horse's gallop, all four hooves were ever off the ground at the same time $[36]$.

little scientific value. And as we need now to capture the series of flow phenomena at the timescale of the process, here is where high-speed imaging comes into play.

High-speed imaging in flow visualization is primarily aimed at obtaining precise information about the position and dimensions of the fluid flow at a series of instants in time, i.e. to resolve to the best possible extent the spatial and temporal scales. High-speed imaging has been key to a series of discoveries and to put the recent high-speed imaging technologies in historic perspective we give a brief overview of the history of high-speed imaging. It all started with the pioneering work of Eadweard Muybridge [36] and Etienne-Jules Marey [37,38] who built the first framing cameras in the late 19th century (1860-1880). This was long before motion picture film was patented in 1894. Muybridge's challenge was to settle a long standing debate: "Is there a moment in a horse's gait when all four hooves are off the ground at once?". Muybridge was a pioneer of scientific research in motion analysis. He devised a camera system consisting of 12 individual negative film cameras, each triggered milliseconds after each other, to collect a series of motion pictures, see Fig. 2. Muybridge's multi-camera concept was also applied in the Manhattan project. Berlyn Brixner [39] at Los Alamos used a set of 37 synchronized cameras in a row, all recording at the conventional 30 frames per second, to record the first nuclear explosion at $1 \mathrm{~ms}$ time interval. With the need for ever increasing recording speeds, rotating mirror technology was introduced for ultra high-speed imaging for thermonuclear weapons research in the 1950s. The first ultra-high-speed rotating mirror camera was constructed, capable of recording at 1 million frames per second (Mfps). The incoming image was spinned around by rotating prisms or mirrors along the inside of a 1-m radius arc covered with a static negative 


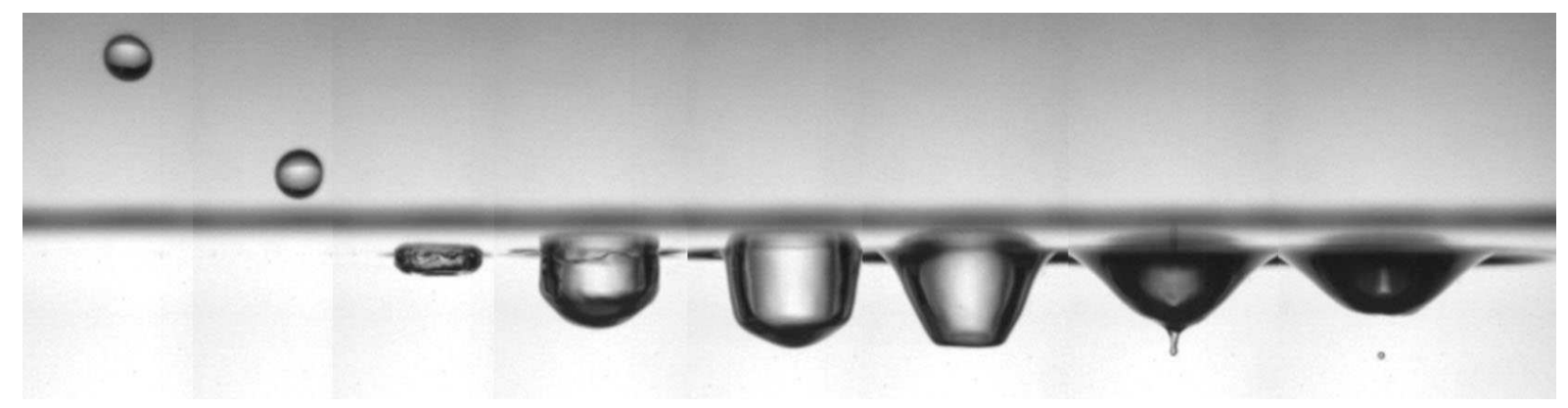

Fig. 3 A 3-mm droplet impacting on a water surface creates a cavity, which collapses due to hydrostatic pressure. It forms the well-known Worthington jet upward [34], not shown here. The downward jet pinches off a small bubble. It is this tiny bubble that produces the sound of falling rain [44] (movie online).

film rail. Using a very clever optical configuration, known as the Miller principle [40], the experiments were photographed, and the pictures revealed timing errors in detonation which neither the ballistics engineers nor the physicists had anticipated. This was the last major technical hurdle of this history-changing project, solved by the ultra high-speed camera. A series of rotating prisms, rotating mirror, streak cameras and rotating drum cameras were developed in the years to follow, see overviews in $[41,42]$. All systems in those days relied on negative film and for many reasons these have now been replaced, first by VHS and Video 8 in the 1980s, followed by digital CCD and CMOS units after the turn of the millennium. In the race for the need for speed the mechanically driven cameras were superseded by electro-optical camera systems, see the overview in [43], notably the image converter cameras. These cameras are a modified version of the Gen I image intensifier tubes where the beam of photoelectrons, formed at a high-gain photocathode, were rapidly deflected such that multiple images could be projected separately onto a phosphor screen, where it was photographed and in later years imaged onto a CCD imager. Image converter cameras were commercialized as Imacon cameras by Hadland, and similar models were offered by Hamamatsu and Cordin.

Digital high-speed imaging systems are available today from 200 frames per second to 200 million frames per second. Systems vary from compact handheld consumer-type cameras to systems filling an entire lab. Two classes of imaging systems are common. First, systems with a single CCD and CMOS chip. These systems are capable of acquiring 1,000-5,000 frames per second at up to 1080p HD-TV resolution, which also makes them highly popular for sports and nature and science documentaries. Many of these systems can achieve higher frame rates by rapidly shifting the charge to neighboring cells in the chip at the expense of a considerable reduction in resolution, e.g. $128 \times 48$ pixels at $500 \mathrm{kfps}$ for a typical high-end camera. This obviously limits the use of these type of cameras for frame rates exceeding one million frames per second; one ends up with only a couple of pixels. That is why the second class of imaging systems makes use of the old concepts of Muybridge and Brixner, i.e. 
if one cannot solve the problem with one camera, one simply uses more. The number of frames for a sequential recording is then dictated by the number of cameras available in the camera system, e.g. 128 frames for the Brandaris camera [45] running at 25 Mfps and eight frames for the highest imaging frame rates of 200 Mfps for the image converter cameras [46], such as the Imacon-200. But the limitations of high-speed imaging systems are not only in the frame rate and the number of frames. Selection criteria for the use or the choice of a particular high-speed imaging system also include a detailed assessment of the system's sensitivity, actual (!) resolution, pixel sizes, trigger options, shutter speeds, the size or weight, and of course cost.

A typical recording with a digital high-speed camera is shown in Fig. 3. It shows how a droplet impacts on a pool of water, forming a cavity that collapses. A thin microjet is formed, on the axis of the collapsing cavity, pointing downward, and a microbubble with a radius of the order $100 \mu \mathrm{m}$ pinches off from the jet. From synchronous hydrophone measurements we identify that the familiar sound of dripping water is directly associated with the formation of this bubble, not with the contact of the droplet with the water surface [44]. The sound is harmonic and is related to the size of the microbubble formed through its eigenfrequency $f_{0}=3.3 \mathrm{mmkHz} / R_{0}$ [47]. And since the droplet impact formation changes from droplet to droplet, also the bubble formation process changes from droplet to droplet, leading to the characteristic "plik, plok, pluk" sound (audio on-line).

This particular series of events was recorded at 1,000 frames per second, with an exposure time setting of $150 \mu \mathrm{s}$. But what dictates the frame rate and what dictates the exposure time? What is it in this dripping water problem, and in flow visualization in general, that we are after? What is it that we want to investigate? Is it the impact of the droplet or the formation and inertial dynamics of the cavity? Is it the microjet formation, the microbubble pinch-off, or the microbubble oscillations leading to sound formation? The answer is that we need to know the relevant timescale of the problem; in other words: the flow problem dictates how the high-speed imaging needs to be done. And therefore, the purpose of this review is to provide insight in high-speed imaging technology and to get acquainted with the various high-speed imaging techniques. It will offer basic assessment of the high-speed imaging protocols; first flow problem-oriented, then imaging hardware-oriented. We offer rule of thumbs and we discuss common pitfalls. We will discuss ultra high-speed imaging, at timescales shorter than a microsecond, important for microfluidic applications. We discuss high brightness illumination techniques and offer ways to calibrate and test high-speed imaging systems. And finally we conclude with unique combinations of traditional experimental imaging methods in fluid mechanics (Schlieren, PTV and PIV, fluorescence) with high-speed imaging techniques, opening up a wealth of new opportunities for experiments in fluids. 


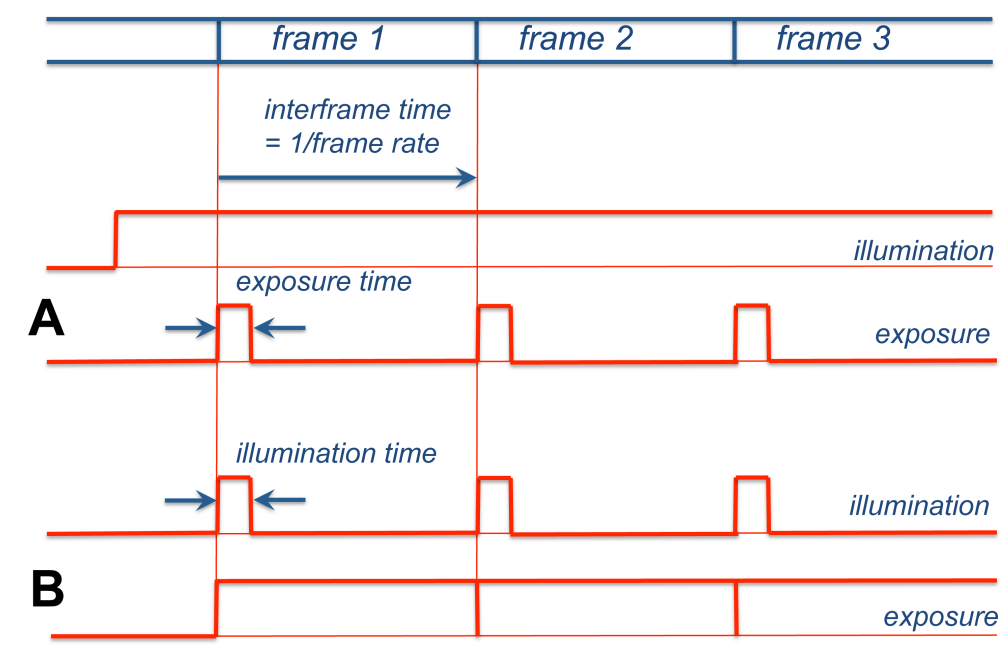

Fig. 4 Schematic representation of the definitions of frame rate, interframe time, exposure time and illumination time in a series of successive frames. A: Recording with continuous illumination and with an exposure time shorter than the interframe time, to freeze the motion to minimize motion blur. B: Pulsed illumination defines the effective exposure. The exposure time can be set arbitrarily, even as long as the interframe time.

\section{High-speed imaging basics}

\subsection{Definitions}

With reference to Fig. 4 we start by defining a set of keywords associated with high-speed imaging. First of all, the frame rate or recording speed is defined as the number of frames taken per second. The units are given in frames per second and is abbreviated with fps. Engineering notation is used to further shorten the units. 1 kfps represents 1,000 frames per second; 1 Mfps represents 1 million frames per second. Normal video rate CCD cameras record at a frame rate of $25 \mathrm{fps}$ (PAL in Europe) or $30 \mathrm{fps}$ (NTSC in US and Japan), typical commercially available high-speed cameras record at a frame rate of 1 to $5 \mathrm{kfps}$. Each frame of a regular video rate camera consist of an interlaced image composed of odd and even fields, a technology dating back from the television standard and an important technique of improving the picture quality of a video signal on CRT devices without consuming extra bandwidth. The odd fields fill the odd rows, the even fields fill the even rows and each field is taken sequentially at a time interval of $20 \mathrm{~ms}$ for PAL and $16.7 \mathrm{~ms}$ for NTSC. For high-speed events, interlacing causes motion artifacts and aliasing, as the frame is composed of two fields taken at different times. Most high-speed imaging systems therefore use progressive scanning, where each field is a complete frame.

The interframe time is defined as the time in seconds between two successive frames and the interframe time is therefore the reciprocal function of the frame rate. An ultra high-speed camera accommodating a set of intensified CCD cameras can have an interframe time of $5 \mathrm{~ns}$, hence it achieves a frame rate of 200 Mfps. 
The exposure time is the duration in seconds that the frame is exposed to the object. The exposure time is typically controlled by a mechanical or electronic shutter. For optimum temporal resolution the exposure time should be equal or smaller than the interframe time. To reduce motion blur the exposure time is typically chosen to be shorter than the interframe time. Some camera systems do not allow to set the exposure time independently from the the interframe time, so for these systems the only other way to control the exposure, is to control the pulse length of the illumination source, e.g. by using a pulsed Xenon flash or a light-emitting diode (LED). In the following we will refer to it as the illumination time, see also Fig. 4B.

Now, the important question we have to ask ourselves before we do the high-speed imaging experiment is:

(a) What frame rate is required?

(b) What exposure time is required?

(c) How do these relate to the magnification?

We should thereby keep in mind that the criterion is quite different for a pure visualization and for illustrative purposes rather than for a quantitative evaluation of the high-speed event.

\subsection{Frame rate}

To answer the first question we will need to find out the relevant time scale of the high-speed event. In some applications we look at a cyclic event of which we know the (driving) frequency. Then according to the Nyquist sampling theorem the required sampling rate or frame rate should be more than twice, but conveniently at ten times the event frequency. In many applications we have little clue of the relevant timescale. One may be inclined to rely on the typical velocity as a guide of the relevant timescale, however, you'll easily be mislead as the time scale also scales with the inverse length scale.

The optimum frame rate $f$ can be estimated from:

$$
f=\frac{N \cdot u}{l}
$$

where $N$ represents the required number of samples, as discussed above a minimum value of 2 , but a typical value of 5-10 would be more appropriate, $u$ a typical velocity and $l$ a typical length scale. Eq. (1) reveals the importance of high-speed imaging in microfluidic applications, where $l$ is small and consequently $f$ is large. 


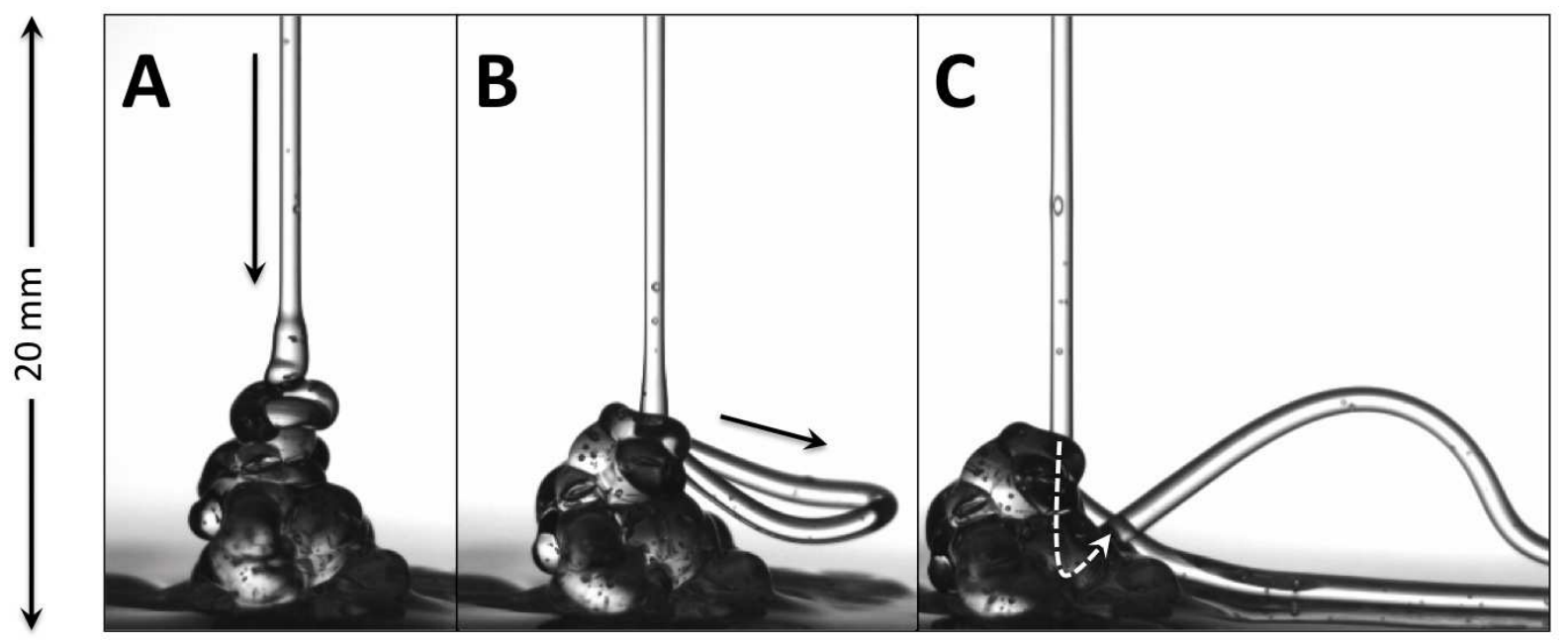

Fig. 5 A series of three frames taken from a high-speed video of leaping shampoo [48]. The shampoo first forms a heap (A) and then displays the Kaye effect [49], where a streamer shoots off in an arbitrary direction (B,C). High-speed imaging of the process has revealed the importance of the non-Newtonian behavior of the shampoo (movie online).

Similarly one should realize that a velocity of $1 \mathrm{~m} / \mathrm{s}$ corresponds to $1 \mu \mathrm{m} / \mu \mathrm{s}$, which means that looking at $\mu \mathrm{m}$ length scales requires $\mu$ s interframe times at these velocities, corresponding to Mfps frame rates.

To exemplify the selection of the proper frame rate we first refer to Fig. 5 showing the Kaye effect $[49,50]$. Here a thin stream of shampoo is poured from a height of $20 \mathrm{~cm}$. The shampoo forms a viscous heap and once in a while a thin jet of shampoo leaps from the heap. The timescale of this problem is governed by the length scale $l$ of the incoming jet, which is of the order of $2 \mathrm{~cm}$, and by its velocity. The velocity $u$ can be estimated from a balance of the potential energy $\dot{m} g h$ and the kinetic energy $\frac{1}{2} \dot{m} u^{2}$, leading to $u=\sqrt{2 g h}$. With a height of $20 \mathrm{~cm}$, we obtain an estimated velocity $u=2 \mathrm{~m} / \mathrm{s}$, independent of the mass flow rate $\dot{m}$. Using Eq. (1) we then find that the leaping shampoo experiment zoomed in at the heap (field of view of $20 \mathrm{~mm}$ ) can be visualized with a frame rate of order $1 \mathrm{kfps}$. Tracking of the microbubbles entrained in the incoming stream confirm the impact velocity. Tracking of the very same microbubbles in the outgoing stream, see Fig. $5 \mathrm{C}$ reveal the energy dissipation in the dimple and were key to our understanding of this problem [48]: at some instant, due to a favorable geometry, the incoming jet will slip away from the heap and while for a viscous Newtonian fluid such a slip would only lead to a small disturbance in the wrinkling or coiling pattern, in the shear-thinning shampoo the resulting high shear rate forms a low viscosity interface leading to an expelled jet at low inclination. Meanwhile, the incoming jet will exert a vertical force on the viscous heap forming a dimple. The dimple deepens because of the sustained force exerted onto it by the incoming jet thereby erecting the outward going jet. The inclination of the streamer steepens until it hits the incoming jet and disturbs or even interrupts the in-flow, thereby halting the Kaye effect. 

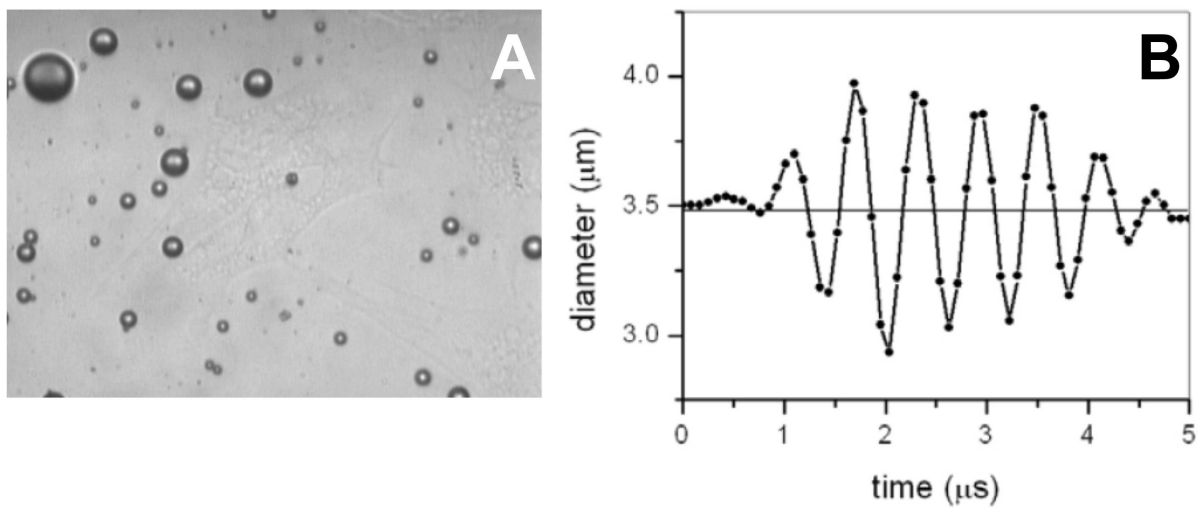

Fig. 6 Microbubbles with a radius $1-10 \mu \mathrm{m}$ (left) driven by an 8-cycle ultrasound pulse at a frequency of 1 MHz are imaged at a speed near 10 million frames per second. From the contour of the bubbles we can deduce the radius-time curve (right), which helps to model the dynamics and nonlinear behavior of these microbubbles for contrast-enhanced ultrasound imaging (movie online).

As a second example we now look at oscillating microbubbles, see Fig. 6, used as contrast bubbles in medical ultrasound imaging. The bubbles have a typical size of 1-5 $\mu \mathrm{m}$, they are coated by a stabilizing phospholipid shell, and the bubbles are driven near resonance [47] by an ultrasound pulse with a typical frequency of 1$5 \mathrm{MHz}$. The bubbles generate an echo which is picked up in the far-field in pulse-echo mode by a transducer. While the surrounding tissue reflects the ultrasound linearly, the nonlinear bubble dynamics leads to a harmonic contribution, which by picking up the harmonics leads to the contrast with the tissue. And as blood is a very poor ultrasound scatterer, the injection of these microbubbles facilitates the real-time perfusion imaging of organs. From potential flow arguments the nonlinear bubble echo pressure $P_{s}$ at a distance $r$ can be calculated directly from the bubble oscillations: $P_{s}=\rho R\left(R \ddot{R}+2 \dot{R}^{2}\right) / r^{2}$, with $R$ the radius of the bubble, $\dot{R}$ the velocity of the bubble wall, and $\ddot{R}$ the acceleration of the bubble wall. To understand and to optimize contrast-enhanced medical ultrasound imaging it is therefore fundamental to record the bubble dynamics with sufficiently high accuracy as to be able to calculate its time derivatives, i.e. velocity and acceleration, for non-linear echo pressure evaluation. Here the system is governed by the driving frequency of $1 \mathrm{MHz}$ and thus the sampling frame rate must be of the order of $5 \mathrm{Mfps}$ for the velocity, and another factor 5 higher for the acceleration, $25 \mathrm{Mfps}$. It will be shown later how such a high imaging frame rate can be achieved. Note that in this particular example of vibrating microbubbles, the relative amplitude of oscillation $\Delta R / R_{0}$ is of the order of $10 \%$ of the ambient radius $R$. With $R=R_{0}+\Delta R \sin \omega t$ we find that the velocity of the bubble wall $\dot{R} \simeq \omega \Delta R \cos \omega t$ is of order $1 \mathrm{~m} / \mathrm{s}$, the same as for the leaping shampoo. So again, velocity is not the governing factor for the frame rate determination, but must rather be combined with the length scale of the problem. 


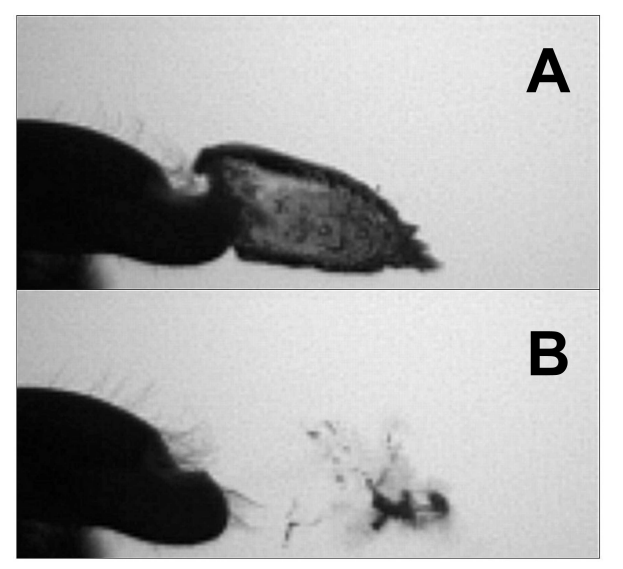

Fig. 7 A pistol shrimp produces a cavitation bubble (A) in a high-speed water jet formed after claw closure, followed by inertial collapse (B) producing a loud snap and a shockwave that stuns. Taken from Versluis et al. [4] (movie online).

\subsection{Exposure time}

To answer the second question (on the exposure time) let us first make the remark that for many applications we do not need a high frame rate per se, however for delineation purposes and for sufficient spatial resolution for scientific analysis it is advantageous to reduce the exposure time to freeze the motion. Fig. 7 for example shows the collapse of a cavitation bubble produced by the snapping shrimp [4]. The shrimp closes its snapper claw with lightning speed and water is squeezed out from between the claws. It forms a jet with a velocity of $25 \mathrm{~m} / \mathrm{s}$, fast enough to create a cavitation bubble due to the reduced pressure of the jet, which is below the vapor pressure of water. The cavitation bubble grows to a size of several millimeters, followed by an inertial collapse, see Fig. 7B. At a frame rate of $2 \mathrm{kfps}$ the interframe time is $500 \mu \mathrm{s}$, the exposure time here is set at $25 \mu \mathrm{s}$. The cavitation bubble exists only for $300 \mu \mathrm{s}$ or so; if the exposure time would have been set to the interframe time the bubble dynamics would be completely smeared out and the bubble would probably not even be visible. With the current recording settings very small details of the collapsed bubble cloud are captured, see again Fig. 7B. If we were to resolve the full dynamics of the bubble collapse then we would have to record at a reference timescale of the order of the exposure time used here (as this freezes the motion). A $25 \mu$ s interframe time then corresponds to a frame rate of $40 \mathrm{kfps}$, see the details in [4].

In a similar way we image, in a granular fluid, the impact of a steel ball into loose very fine sand, see Fig. 8. It creates a splash, followed by an intense jet with a height that exceeds the release height of the ball [51]. The frame rate was 2,000 frames per second, the exposure time was set much smaller to capture the fine details of the grainy splash, the jet and the clustering of particles within the jet. 


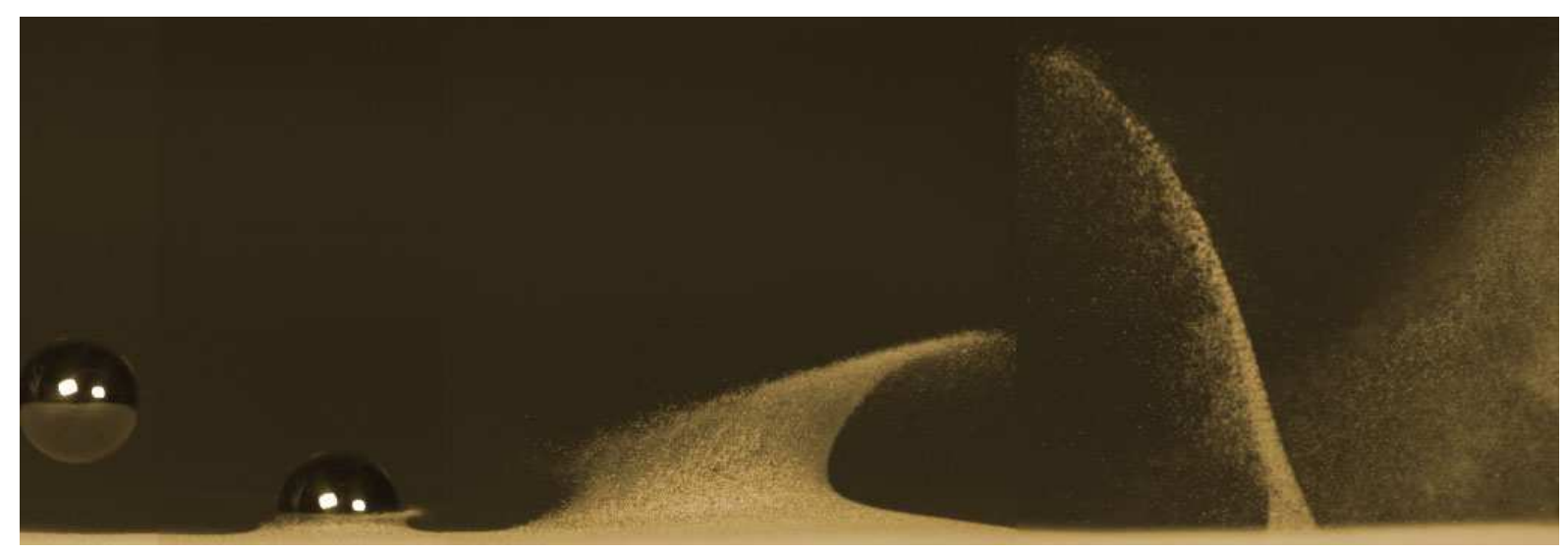

Fig. 8 An oblique impact of a steel ball into fine very loose sand creates a splash followed by a jet in the backward direction. The jet is a result of the collapse of a cylindrical void created in the sand upon impact [51] (movie online).

A shorter exposure time always goes at the expense of the signal level. It decreases linearly with the exposure time setting, therefore it must be compensated by an increased illumination level. Going from video rate imaging to high-speed imaging sometimes requires an increased illumination level of four orders of magnitude, which is not easily overcome; therefore, illumination considerations are included in detail in this review. The exposure time can be as short as $1 \mu$ s for a CCD camera using an electronic shutter. Specialized double-frame particle imaging velocimetry (PIV) cameras have an exposure time of $200 \mathrm{~ns}$, while an intensified CCD camera can have an exposure time as short as $250 \mathrm{ps}$ owing to the fast electronic gating of the image intensifier.

The exposure time can be set in several ways. The simplest way to control the exposure time is by using a mechanical shutter. The fastest mechanical shutters open and close in just under $1 \mathrm{~ms}$. A shorter exposure time can be achieved by controlling the opening and closing phase in separate shutters, for instance the start of the exposure can be initiated by the perforation of a thin metal foil, while the end of the exposure time can be controlled by redirecting or removing a mirror. In some systems we find liquid crystal shutters. They can be switched down to milliseconds timescales, but the transmission can be poor for an open shutter (50\%), and the faster shutters lack sufficient contrast ratio and light bleeds through in the 'closed' position.

In $\mathrm{CCD}$ and CMOS cameras the exposure time is set by an electronic shutter that can be set manually (by DIP switches), electronically or by software. Shutter times can be as short as $1 \mu$ s. Even shorter exposure times can be accomplished by using an image intensifier, which converts photons imaged onto a photocathode to electrons. The electrons are accelerated onto a multi-channel glass plate (MCP), which amplifies the electrons by 4 to 5 orders of magnitude. Double-MCP configurations are used to achieve even higher gains. The amplified electrons then impinge on a phosphor screen to convert them back into photons. Image transfer is conserved 

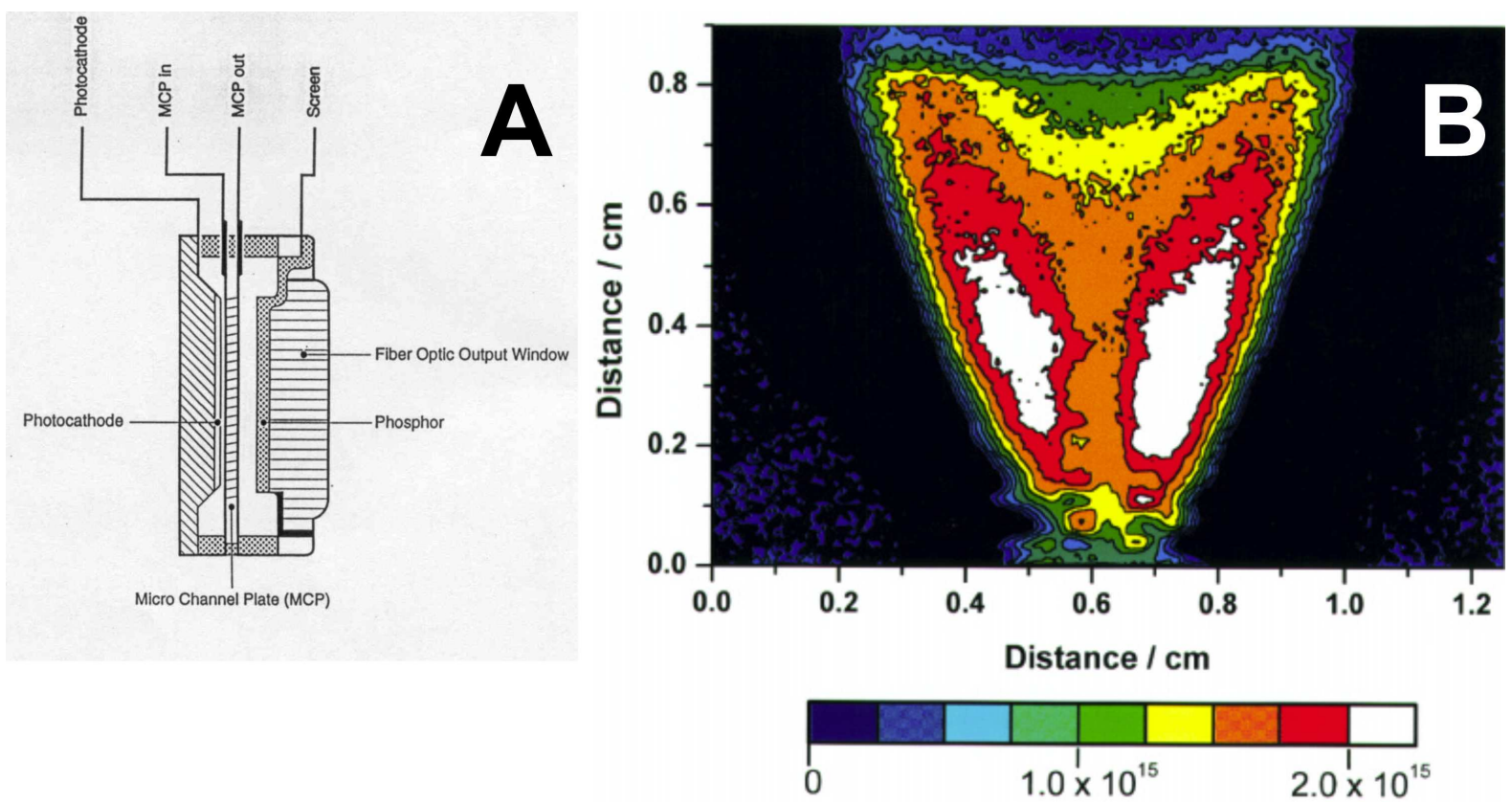

Fig. 9 A: Schematic cross section of an image intensifier. Photons impinge from the left and are converted to electrons by the photocathode. Multiplication in the multichannel plates (MCPs) amplifies the number of electrons by a factor $10^{5}$. The electrons hit the phosphor screen where they are converted back to photons. B: A single-shot quantitative planar image of the hydroxyl $\mathrm{OH}$ radical distribution in a premixed methane/oxygen flame using planar laser-induced fluorescence taken with an image-intensified CCD camera with an exposure time of $10 \mathrm{~ns}$ [52].

through the image intensifier at the expense of loss of resolution and the addition of noise, in particular for higher gain settings. A fiber-optic taper couples the phosphor screen onto a CCD chip for recording, see Fig. 9A. The advantage of using the image intensifier is three-fold. First, as the name implies, it intensifies the image. Secondly, and most important for our interest here, is the ability to gate the exposure time, both the timing and the duration. By switching the voltage between the photocathode and the MCP the electrons can be either accelerated or repelled from the entering the MCP; switching of the $250 \mathrm{~V}$ can be accomplished in 5 ns. And finally, as the phosphorescence of the phosphor screen decays slowly (order milliseconds for a P-43 phosphor) it gives us the ability to capture fast events at nanoseconds timescale with a CCD with a relatively long video-rate read-out time of 16.7 or $20 \mathrm{~ms}$. For some fluorescence applications the image intensifier is also a convenient tool to transform ultraviolet photons, which cannot be detected with a silicon-based chip, to photons in the visible, blue or green, depending on the phosphor-type.

\subsection{Magnification}

Now to answer the quantitative measure of the exposure time setting, we remind us that the application of high-speed imaging in flow visualization is primarily aimed at obtaining precise information about the position 
and dimensions of the fluid flow at a series of instants in time. It is therefore of prime importance to capture the smallest details in both temporal and spatial resolution at high contrast. With the previous sections in mind we can now quantify the criteria to achieve optimum high-speed flow visualization. These are

(i) to fulfill the spatial Nyquist criterion,

(ii) to maximize the signal-to-noise ratio at the sensor,

(iii) to minimize motion blur.

Assuming that an imaging objective lens with the highest possible numerical aperture is chosen for the experiment, the effective optical magnification $M$ must be sufficient to avoid undersampling of the image with respect to the spatial resolution, $R_{s}$, as defined by the Rayleigh criterion [53]. The spatial Nyquist criterion requires that at least 2 pixels lay within $M \cdot R_{s}$. Hence it follows that the magnification should be such that:

$$
M>2 r_{p} / R_{s}
$$

where $r_{p}$ is the pixel size of the sensor located in the image plane. Larger magnifications allow denser spatial sampling (known as oversampling), but also result in a smaller field-of-view and lower image brightness. The magnification is therefore limited by the optimum size of the object image and the available illumination intensity.

To ensure maximum image contrast the intensity of the illumination should be adjusted to cover the full dynamic range of the camera sensor. The extent to which the intensity can be varied is, however, limited due to the reciprocal relationship between illumination intensity and exposure time. To clarify, an image with a certain fixed brightness can be obtained with either low light conditions and longer exposure times, or, vice-versa, a bright flash and short exposure. In the situation where an object is motionless both settings result in identical images. However if the object of interest is moving, the image becomes susceptible to motion-blur. This undesired effect causes a smeared appearance of the image of the object due to its displacement during the time the image is recorded. Minimizing motion-blur constitutes the third criterion that would be required to accurately capture a single high-resolution image of a moving object and this can be achieved by adjusting the temporal resolution of the imaging system. As discussed before, this temporal resolution is determined by the camera exposure time $\tau_{c}$ or by the illumination time $\tau_{p}$. Evidently, the actual temporal resolution $\tau$ will be the shortest of both durations, hence $\tau=\min \left(\tau_{p}, \tau_{c}\right)$. If we now define $\epsilon$ as being the displacement in pixels of the object moving with velocity 

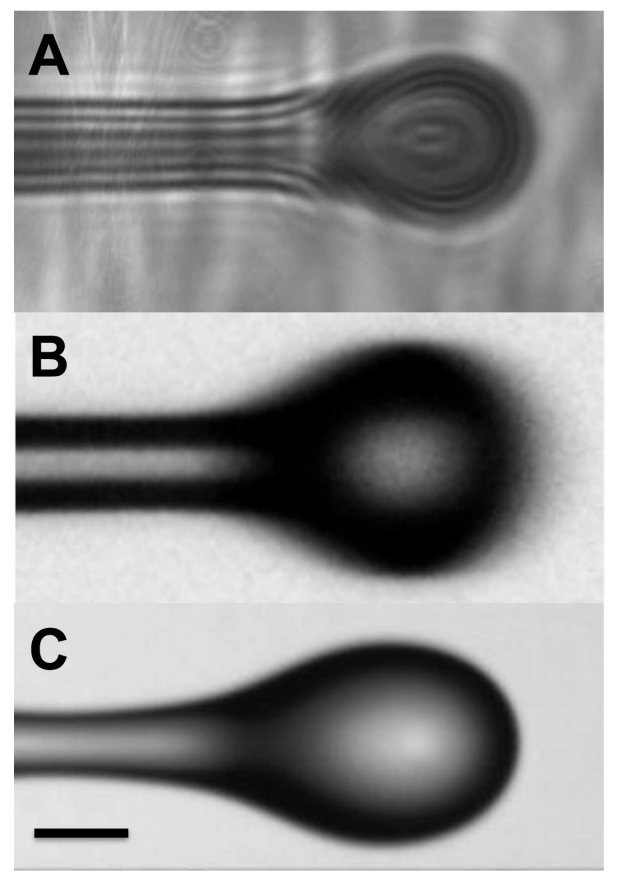

Fig. 10 A snapshot of the head of a single inkjet droplet fired from a drop-on-demand (DOD)nozzle system. The DOD frequency is $100 \mathrm{kHz}$ and the speed of the droplet is near $20 \mathrm{~m} / \mathrm{s}$. The scale bar denotes $10 \mu \mathrm{m}$. A: Image obtained by a 7-ns laser flash illumination. Coherence of the laser light leads to optical fringes and speckle formation. B: 300-ns LED illumination reveals motion blur as a result of the relatively long illumination time. C: The motion artifact is reduced by a 7-ns illumination with a laser-induced fluorescence pulse using iLIF [54].

$u$ during time $\tau: \epsilon=\tau M u / r_{p}$ motion-blur will be minimal if $\epsilon \leq 1$. In most experiments the object velocity, the magnification and the pixel size are predetermined so motion-blur is avoided by choosing an exposure time or illumination time such that:

$$
\tau \leq r_{p} / M u
$$

Drop formation in inkjet printing is an almost perfectly reproducible process, and single flash photography is frequently applied for its visualization [55,56], see Fig. 10A. Here we demonstrate the use of two different flash illumination sources to capture the front end (or head) of an inkjet microdroplet of $100 \mathrm{pL}$ in volume and with a velocity $u$ of about $20 \mathrm{~m} / \mathrm{s}$. The printhead is an experimental prototype developed by Océ Technologies B.V., similar to the printheads used in references $[57,58]$. The printhead ejects droplets with a diameter of $30 \mu \mathrm{m}$ at a typical velocity of $20 \mathrm{~m} / \mathrm{s}$ during drop formation. Some $35 \mu$ s later the tip of the droplet reaches a terminal velocity close to $6 \mathrm{~m} / \mathrm{s}$. We use a high-intensity Luxeon LED or a HSPS Nanolite KL-L for illumination with pulse durations of $\tau_{p}=400 \mathrm{~ns}$ and $\tau_{p}=16 \mathrm{~ns}$, respectively. The incident light is focused through a collimating lens onto the region of interest. The collimation lens was chosen such that the numerical aperture (NA) matches with that of the microscope objective ensuring maximal optical resolution $\left(R_{s} \approx 2 \mu \mathrm{m}\right)$ and intensity [53]. The microscope (Olympus BX-30MF) is equipped with a long working distance (WD) 20× objective (Olympus, WD 
$25 \mathrm{~mm} / \mathrm{NA}=0.25)$. The camera is a Lumenera LM165 with a sensitive Sony EXview HAD CCD sensor with a pixel size of $6.5 \times 6.5 \mathrm{\mu m}^{2}$, and a resolution of $1392 \times 1040$ pixels. The scale factor for the camera and the $20 \times$ microscope objective combination is $320 \mathrm{~nm} /$ pixel. Fig. 10B and $\mathrm{C}$ show the results of the microdroplet head after it has just exited the nozzle and has slowed down to its terminal velocity. The practical advantages of the combination of high-intensity and short pulses of light for imaging of fast phenomena become apparent from a detailed comparison of the images in Fig. 10. Using Eq. (3) for the indicated magnifications we find that the droplet can only be imaged without motion blur if the illumination times are smaller than $\tau=16$ ns. The droplet displacement during exposure $\epsilon$ is acceptable for the Nanolite, Fig. 10C, however the pulse length of the LED is too long, resulting in motion-blur, as can readily be seen in Fig. 10B.

2.5 Scale and scaling issues

As a final example we now present two cases that relate to the problem of scaling. These include an example on a finite-time singularity and a typical example on how scaling affects high-speed imaging analysis.

Fig. 11A shows the discharge of a liquid from a microscopic nozzle at a sufficiently large velocity and which leads to a continuous jet that breaks up into droplets, so-called Rayleigh breakup, due to the interfacial capillary forces $[59,60]$. Here we can estimate the required frame rate for the formation of microdroplets directly from the capillary breakup time $\tau_{b}=\sqrt{\rho r_{0}^{3} / \gamma}$. With a jet radius $r_{0}$ of $20 \mu \mathrm{m}$, a density $\rho$ of $1 \times 10^{3} \mathrm{~kg} / \mathrm{m}^{3}$ and a surface tension $\gamma$ of $0.072 \mathrm{~N} / \mathrm{m}$, we find $\tau_{b}=10 \mu \mathrm{s}$, which translates to a frame rate of $500 \mathrm{kfps}$. The diminutive Rayleigh jet of Fig. 11B has a radius of $1.25 \mu \mathrm{m}$, and the breakup time scale is reduced to approximately $150 \mathrm{~ns}$, and would therefore require imaging at 50 million frames/s. The frame rate with which Fig. 11B was taken was $13.7 \mathrm{Mfps}$ (interframe time of $73 \mathrm{~ns}$ ) and just barely fulfills the Nyquist theorem.

Fig. 12 shows the collapse of a cylindrical cavity formed after a small disk-shaped plunger is pulled into the water. The collapse is driven by the pressure of the water; near the water surface it is not so high and the collapse speed is relatively low. Near the plunger the water pressure is high, but cavity formation has just started. Consequently, the cavity collapses somewhere in the middle where two jets are formed upon collapse, one upward and one downward. An estimate of the typical length scales and velocities, e.g. those of the plunger: $l=10 \mathrm{~cm}$ and $u=1 \mathrm{~m} / \mathrm{s}$, using Eq. 1 give us a frame rate of $100 \mathrm{fps}$. If we want to study better the moment of collapse, we zoom in, which we know from Eq. 1 inevitably leads to higher frame rates. However, the radius of the neck, $r_{0}$, goes to zero following a universal scaling behavior with $r_{0} \propto\left(t-t_{c}\right)^{\alpha}$, where $t_{c}$ is the time of collapse, and $\left(t-t_{c}\right)$ the 

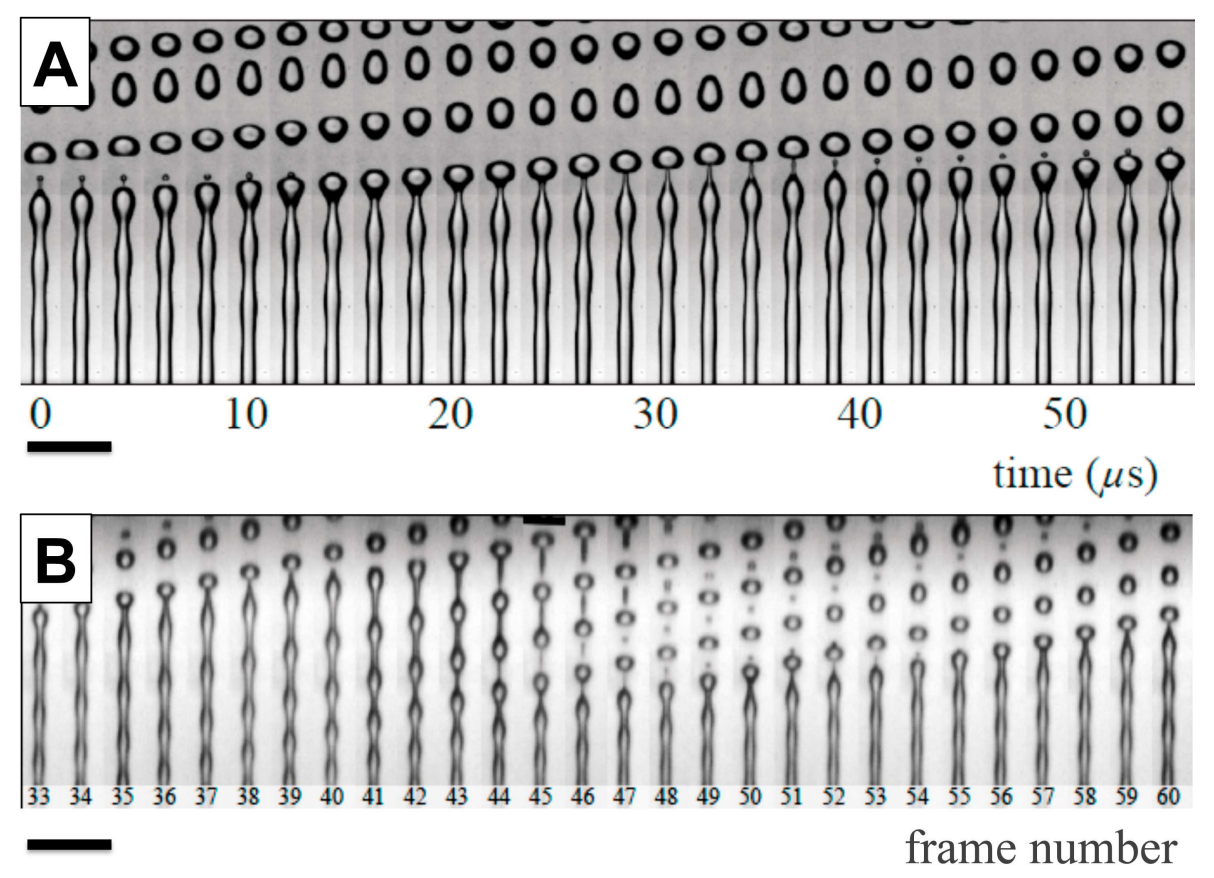

Fig. 11 A: Time series of the high-speed imaging recording at 500,000 fps shows the breakup of a $18.5 \mu \mathrm{m}$ radius jet into a primary droplet and a small satellite droplet [13]. The scale bar in both panels correspond to $200 \mu \mathrm{m}$. A $40 \%$ glycerol-saline solution is supplied at $0.35 \mathrm{~mL} / \mathrm{min}$, equivalent to a jetting velocity of $5.4 \mathrm{~m} / \mathrm{s}$. The scalebar at the bottom right corner denotes $200 \mu \mathrm{m}$ (movie online). B: Time series of the ultra high-speed imaging results recorded at 13.76 Mfps showing the breakup of a $1.25 \mu \mathrm{m}$ liquid jet into droplets. The period in droplet formation is approximately $300 \mathrm{~ns}$. The interframe time is $73 \mathrm{~ns}$. The scale bar in the lower left corner denotes $20 \mu \mathrm{m}$ (movie online).

time until collapse, and where $\alpha$ represents the power law scaling exponent. A simple power law behavior can be predicted based on a purely liquid inertia-driven collapse giving rise to a $1 / 2$ scaling exponent [61,62], although in reality the problem is much more complicated than can be described here [63-65]. The scaling of the neck radius leads to a increasing velocity closer to final collapse, until it reaches infinity, an example of a singularity in finite time, when $t \rightarrow t_{c}$. And while $100 \mathrm{fps}$ was sufficient to capture the dynamics of the cavity collapse at a scale of $10 \mathrm{~cm}$, at a scale of $1 \mathrm{~cm}$ we will need a frame rate of $10 \mathrm{kfps}$, while at a scale of $1 \mathrm{~mm}$ we will need ultra high-speed imaging at a frame rate of $1 \mathrm{Mfps}$. Thus, zooming in leads to a dramatic increase of the high-speed imaging effort.

3 High-speed imaging systems and techniques

\subsection{High-speed photography}

High-speed flow phenomena and small-scale flow phenomena that are typically encountered in microfluidics require both a high spatial resolution and a high temporal resolution. To freeze the motion during exposure we have identified in Sec. 2.4 above that we can employ either a camera with a short exposure time or a light source 

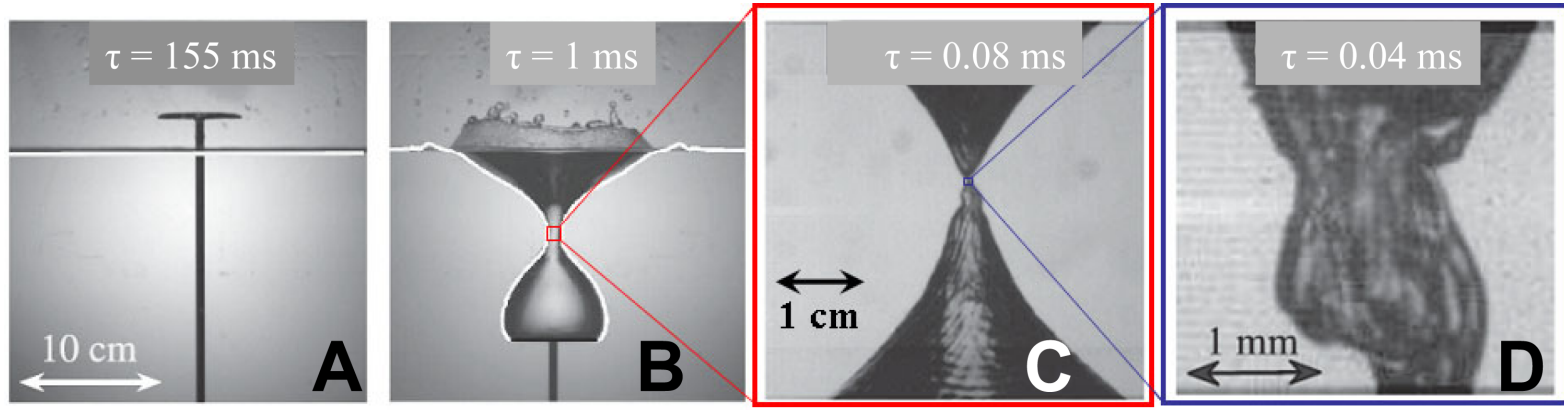

Fig. 12 High-speed imaging of the pinch-off of an air bubble in water shows that the pinch-off is not self-similar. A disk is pulled through a water surface (a) leading to a cylindrical void which collapses leading to pinch-off (b). Since both length and time scales become very small close to collapse, and thanks to the reproducibility of the experiment, this difficulty was overcome by matching several data sets imaged at different frame rates, while zooming into the region around the pinch-off [64] (movie online).

capable of emitting flashes of short duration. The first option generally requires a fast high-speed camera system, which is often limited in its pixel resolution [35], see Sec. 3.3.4. For this reason flash photography is the preferred method for experiments where high-resolution single images are required, since the method does not require a fast shutter and can in principle be performed with any type of camera. Furthermore, the pulse duration of flash illumination sources can easily be shorter than the shortest exposure time of a high-speed camera.

\subsection{Stroboscopic imaging}

We have also identified that once we are interested in real-time flow dynamics we need to resort to imaging at the timescale of the event (which can become as small as nanoseconds in certain cases), with the exception of reproducible and repetitive events. In the latter cases we can use stroboscopic imaging without recourse to the use of an (expensive) ultra high-speed camera system. In stroboscopic imaging we apply the high-speed flash photography technique, and we delay all consecutive recordings by a fixed time spacing $\delta t$, where $\delta t$ is a fraction of the period, $T$, of the event. $\delta t$ is tuned such that it spans the interframe time of the camera, which as mentioned before can in principle be any type of camera, e.g. a CCD video camera or a high-resolution digital SLR photo camera. Playing the captured frames in a video sequence then provides us with a stroboscopic movie of the event, which in many cases is indiscernible from a real-time high-speed movie, but with a dramatically improved spatial image resolution.

Fig. 13 shows the stroboscopic recording of inkjet microdroplets, see the details previously discussed in Sec. 2.4. A short flash was created by exciting a laser dye solution with a pulsed laser [66,54]. The resulting fluorescent light has a very high intensity, while the undesired coherence of the laser light, which would lead to interference and speckle, is removed. The illumination time of the light source is only 7 ns. To record the images 


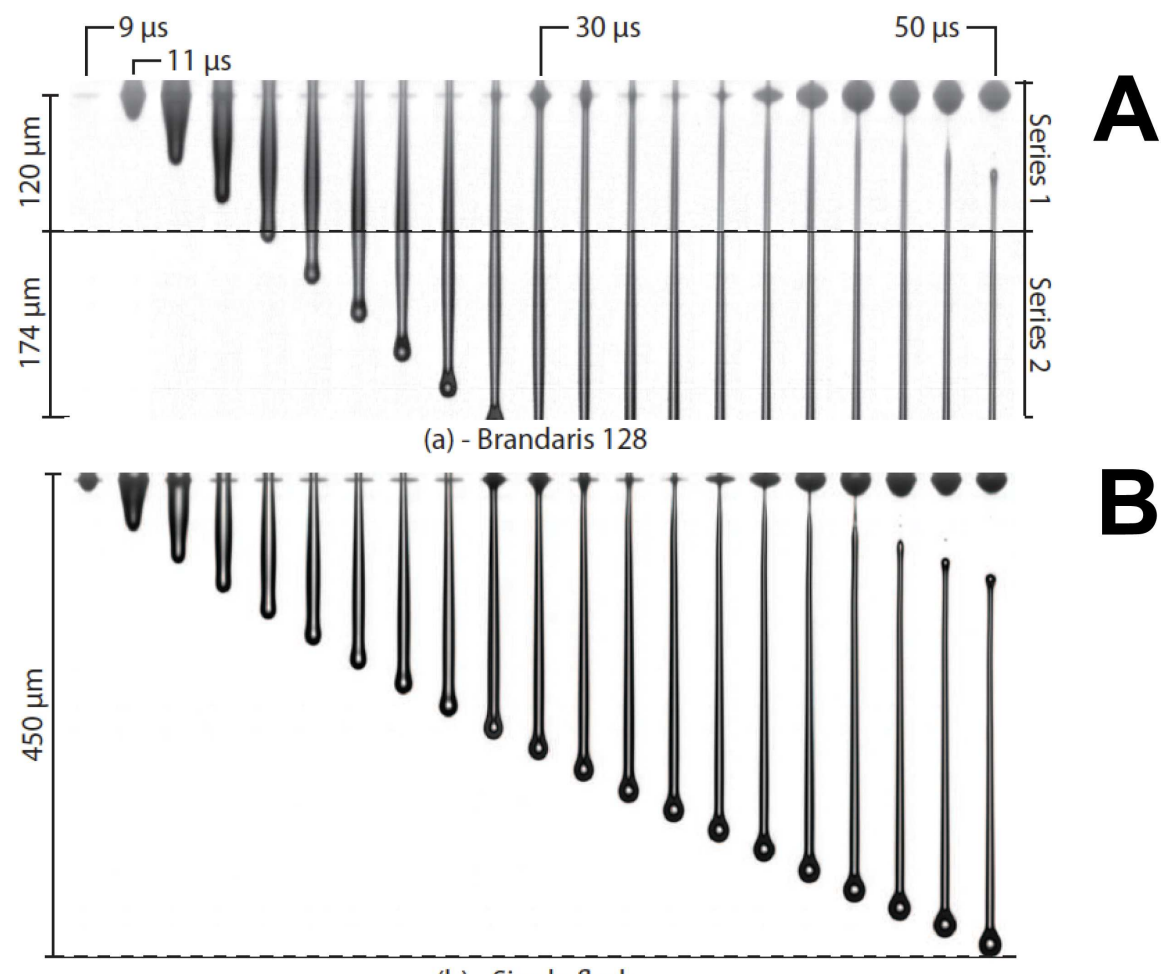

(b) - Single flash

Fig. 13 A selection of images, showing the drop formation originating from a single inkjet channel as a sequence of time. The upper part (A) shows the recordings made with Brandaris-128, while the lower part (B) shows the images captured with the single flash recording. The image illustrates the improved image quality of the single flash recording. Even tiny satellite droplets (radius $<2 \mu \mathrm{m}$ ) can be observed in the tail of the droplet in the last three frames of B (movie online).

a sensitive CCD camera (Lumenera LM165) was used. As an example to estimate the effect of motion blur, based on Eqs. 2 and 3, we list Table 1 where we compare the stroboscopic flash technique and ultra high-speed Brandaris camera. It shows the minimum imaging time scales, $\tau_{\min }$, and the expected motion blur, $\epsilon$, due to the actual time scale, $\tau_{f}$. The combination of the flash light source, the camera and the microscope gives $\epsilon<1$ for the $20 \times$ magnification of the imaging microscope objective; hence, no motion blur is expected.

The short illumination time of the fluorescence light source gives the possibility to choose a very small temporal resolution $\delta t$, corresponding to the smallest timescale in the experiment. In Fig. 13B a step time of 10 ns was used and, to confirm its reproducibility, five images were recorded at each step. To control the delays between the drop, the illumination and the camera, a pulse generator (Berkeley Nucleonics model 565) with a 250 ps precision was used. Additionally, a fully-automated procedure was programmed in Matlab (The MathWorks) source code to control the hardware and to collect the images from the camera. In the experiments the initial phase of the drop was studied, recording only the first $60 \mu$ s of the drop formation. This resulted in a total of 30,000 images for a single experiment, taking about six hours to record. Twenty-one images of the drop evolution are displayed as a time sequence in Figure 13. The high-speed recordings were also performed with the Brandaris-128 ultra 
high-speed camera [45] at a frame rate of 16 Mfps. To illuminate the drop formation, a high-intensity Xenon light source (Perkin Elmer MVS 7010 Xenon flash) was used. Due to the small field of view and the limited number of frames, multiple movies were required to visualize the entire drop formation. The total time of the drop formation is approximately $60 \mu \mathrm{s}$. With an interframe time of $62.5 \mathrm{~ns}$, the Brandaris-128 camera acquires 128 frames in $8 \mu \mathrm{s}$ in a single recording session. Therefore twelve sequential movies must be captured to record the full extent of the drop formation process. The twelve movies were subsequently stitched together into a single continuous movie (which can only be done owing to the near-perfect reproducibility of the inkjet system). In addition, with the small field of view of the Brandaris camera at the $20 \times$ magnification, two series of movies were recorded at different axial distances from the nozzle. The results are displayed in Figure 13A. If, however, the flow phenomenon is not repetitive, or uncontrollable, or unexpected, or when transients are present, in other words, when still photography and stroboscopic methods fail, then there is no other option as to resort to high-speed imaging, which will be the subject of the following section 3.3.1.

\subsection{High-speed imaging}

Fig. 14 gives an overview of commercial and specialized high-speed imaging systems. The left part of the diagram comprises of commercial CCD and CMOS systems with an upper frame rate near 5,000 fps. These systems typically have a large number of pixels allowing detailed and high-resolution high-speed imaging. The center part of the diagram is occupied by high-end systems, roughly from a frame rate of 5,000 fps to 1 Mfps. As the throughput of the imaging system is limited, the number of pixels recorded drops linearly with increasing frame rate. The right part of the diagram contains ultra high-speed camera systems at frame rates exceeding one million frames per second. These include highly specialized image sensors with in-situ storage, digital rotating mirror

Table 1 The experimental parameters for stroboscopic flash recordings and for high-speed imaging. The required minimium imaging time scale $\tau_{\min }$ and the expected motion blur in the experiments, $\epsilon$, was calculated for a velocity of $20 \mathrm{~m} / \mathrm{s}$ and an imaging time scale $\tau_{p}=7 \mathrm{~ns}$ for the single flash recording, and $\tau_{c}=63 \mathrm{~ns}$ for the Brandaris- 128 . The table illustrates the advantage of the flash recording compared to the high-speed recordings; the resolution is higher, the field of view larger, and the actual imaging time scale matches the required one.

\begin{tabular}{|c|c|c|}
\hline & flash & Brandaris \\
\hline \hline Resolution $R_{S}(\mathrm{~nm} /$ pixel $)$ & 320 & 550 \\
\hline Field of view $(\mu \mathrm{m})$ & 449 & 174 \\
\hline min. exposure $\tau_{\min }(n s)$ & 16 & 27 \\
\hline actual exposure $\tau_{p}, \tau_{c}(\mathrm{~ns})$ & 7 & 63 \\
\hline motion blur $\epsilon$ & 0.43 & 2.3 \\
\hline
\end{tabular}




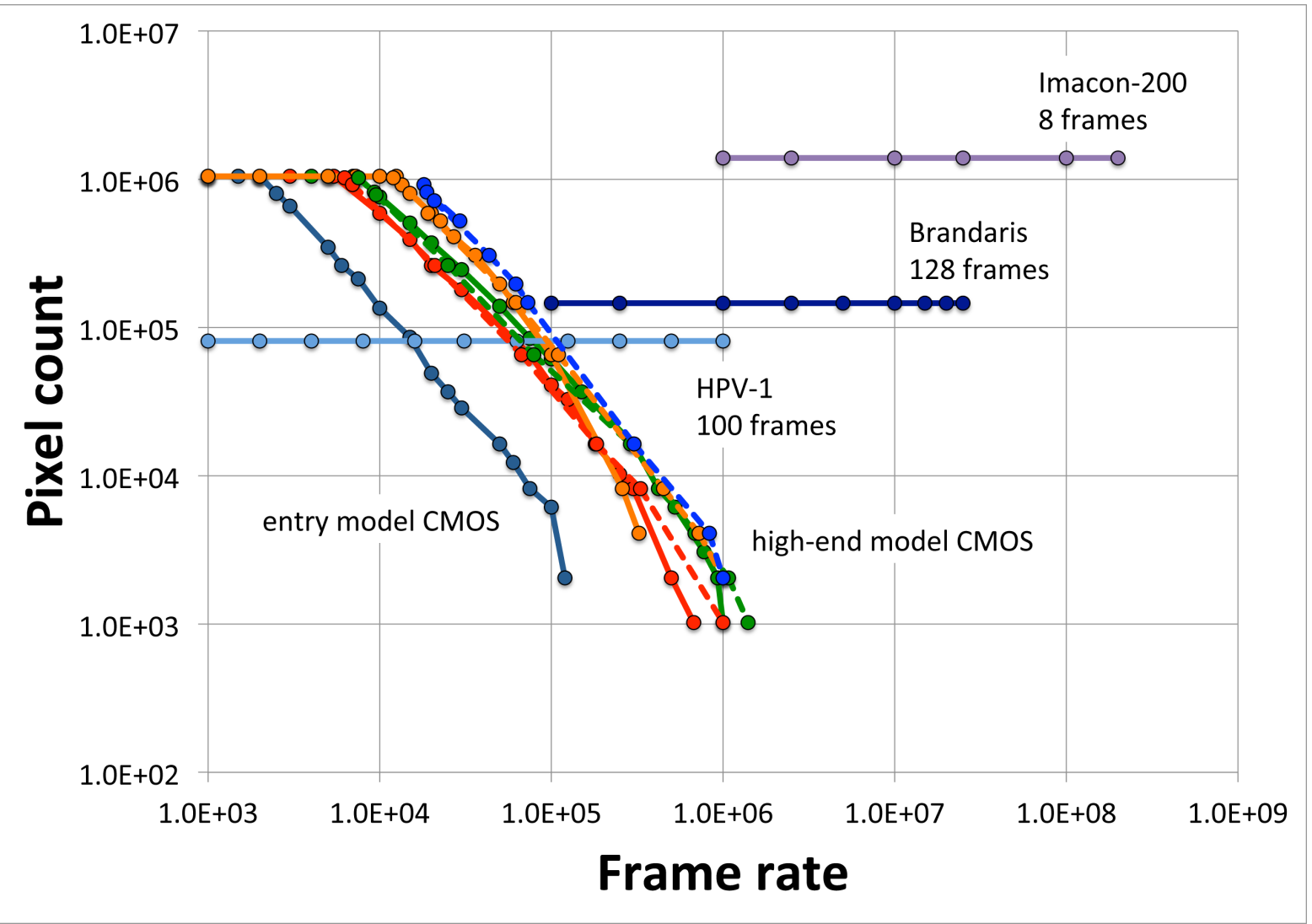

Fig. 14 Overview of a range of high-speed imaging systems. To the left camera systems comprising of a single digital chip with a superb 1 megapixel resolution at 1,000 frames per second. These systems are also capable of recording at hundreds of thousands frames per second at reduced resolution. The second class of camera systems (top right) combine multiple cameras/CCD chips to achieve tens of millions frames per second recordings. These systems have the same image resolution throughout.

systems and image-intensified ultra high-speed imaging devices with frame rates of up to 200 million frames per second.

\subsubsection{CCD and CMOS systems}

Charge coupled device (CCD) chips (Nobel prize in Physics 2009 for Boyle and Smith for the invention of an imaging semiconductor circuit) are silicon semiconductor chips consisting of an array of photo-sensitive picture cells, called pixels. An array is built up of $M$ columns $\times N$ rows of pixels. The incoming photons are converted into a charge (Nobel prize in Physics 1921 for Albert Einstein for the discovery of the photoelectric effect) by integrating the charge of each of the electrons contained in the potential well of the pixel. The deeper the potential well, i.e. the more electrons can be contained in the pixel, the higher the dynamic range of the CCD chip. The dynamic range can be up to 17 -bits deep, i.e. more than $1.3 \times 10^{5}$ separate gray scales can be identified. A row of pixels is read out by shifting the charge into the shift register of the CCD chip. The content of the serial shift 
register is then transferred to the camera circuit board where it is preamplified and digitized. A digital image is thus built up row-by row in the frame buffer of the chip. Read-out of a normal video-rate CCD chip is $20 \mathrm{~ms}$; whereas high-speed imaging CCD chips have a special chip architecture and special A/D conversion architecture with a typical throughput of up to 5 Mpixels per millisecond, which corresponds to several Gigabits per second.

The sensitivity of a CCD chip is coupled to the noise floor within the individual pixels. Cooling reduces the thermal dark noise that collects over time, but for high-speed imaging with only very short read-out times, that is not relevant. Signal noise and read-out noise are intrinsic to the system, and should be carefully assessed. CCD chips can be very sensitive and unintensified CCD cameras are therefore used in surveillance technology. A light level of 0.0005 lux can be detected with a CCD, where 1 lux is already considered sensitive for consumertype handycam CCDs. Pixels can be as small as $2-5 \mu \mathrm{m}$, making up a very compact chip. The pitch of the photosensitive area and the total pixel area along either the row or the column direction does not correspond to the full extent of the photosensitive part. This ratio is called the fill factor. Surface-coated microlens arrays can improve the effective fill factor to give a yield of almost unity. Blooming occurs when the potential well overspills and charge leaks to the neighboring pixels. As many of us use high-speed imaging in back-illumination, anti-blooming architecture is welcomed on-chip, and the microlenses also compensate for the loss of sensitivity as a result of anti-blooming technology, see Fig. 15.

While CCD chips have a better image quality because of their reduced noise component, chips fabricated using the recent Complementary Metal-Oxide Semiconductors (CMOS) technology are by far less expensive, faster and require less power to operate, which makes them popular for webcams an mobile phones. Each CMOS pixel has its own read-out channel and can therefore be read-out individually, and no shifting of the rows is necessary, which dramatically reduces the read-out time of a subframe of the chip. As also the size of the data extracted from the region-of-interest is reduced, CMOS chips allow for a much faster readout, up to 1 million frames per second, before the upper limit of the throughput is reached. CMOS chips are, literally 'a bit', more noisy. Therefore the pixels in CMOS high-speed imaging systems are often larger, typically of a size of $20 \mu \mathrm{m}$, to increase their sensitivity. For microscopic applications this can be a problem as a higher magnification will be needed to obtain a similar field of view.

Color information in CCD and CMOS chip systems is typically obtained by placing a color filter array in front of the chip. The Bayer filter arrangement is the most popular, although different types of filters exist with a modification of colors and arrangement or using dichroic mirrors. Moreover color information can also be obtained 


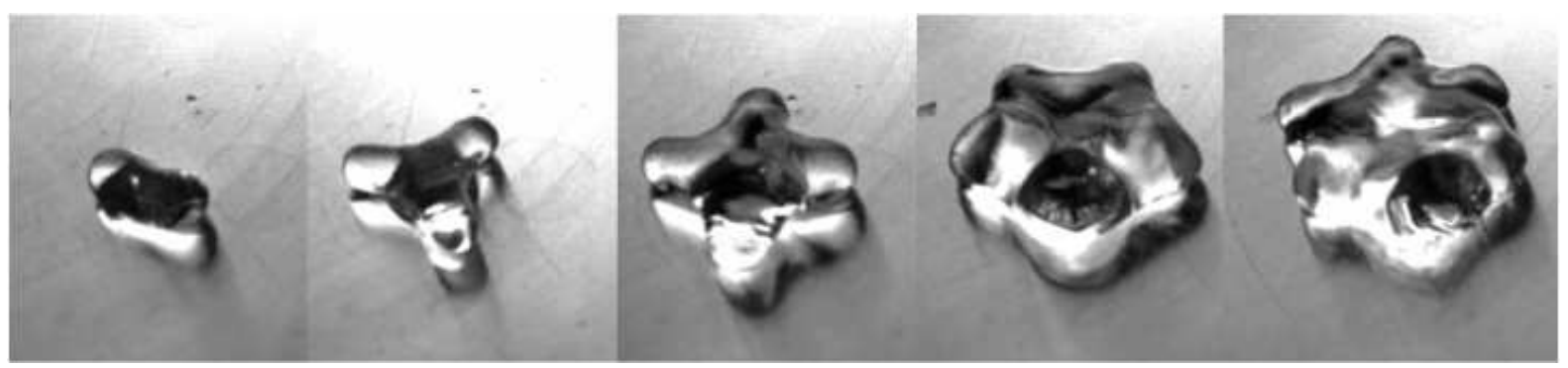

Fig. 15 Surface mode vibrations of a $10-25 \mathrm{~mm}$ droplet on a $220^{\circ} \mathrm{C}$ hot plate. The droplet hovers as a result of the Leidenfrost effect. A surface instability is formed with mode $\mathrm{n}=2,3,4$ etc. and is directly related to the size of the droplet. The anti-blooming of the camera allows for a crisp and clear images with coverage of the full dynamic range (movie online).

with multi-sensor cameras using dichroic and trichroic beam splitter prisms to separate out the colors per sensor.

These systems typically have superior image quality and lower noise levels.

The Bayer filter consists of 4 color filters (red, green, green, blue - RGGB) per set of 4 pixels. An RGB image is recovered by demosaicing algorithms, which interpolate the set of red, green, and blue pixel values for each point. As each filter blocks approximately $2 / 3$ of the incoming light, insertion of the color filter array goes at the expense of a factor 3 in sensitivity. Therefore, if color is not an essential ingredient for the flow visualization, it is recommended to use the monochrome models.

With decreasing interframe and even shorter exposure times, less photons reach the detector. The compromise between frame rate and sensitivity is a recurring problem in high-speed imaging. A similar compromise is found between sensitivity and magnification, which decreases the light collection efficiency quadratically. The solution can be found on either end: on the one hand increase the sensitivity of the detector and on the other hand increase the brightness of the image or the object through more intense illumination or better light collection efficiency.

The sensitivity of the detector is related to the noise level of the detector. Many of the sources of noise are intrinsic to the design of the imaging chip. Consequently an increase of the sensitivity of silicon-based chip technology (CCD or CMOS) is difficult to accomplish. The most sensitive general CCDs are as sensitive as the most sensitive high-speed CCDs. One should therefore keep in mind that a high-speed event that is recorded in video rate at $25 \mathrm{fps}$, once recorded at $100 \mathrm{kfps}$ needs an increased illumination of at least 3 orders of magnitude based on its decreased interframe time, something which is non-trivial with even the brightest flood lights. Also keep in mind that if we would like to keep the field of view identical, we need to magnify by a factor 10 as a result of the reduced pixel count (see Fig. 14), leading to an additional loss of two orders of magnitude. Here, we should in principle revert to pulsed illumination instead, with all energy concentrated in the duration of the exposure. Xenon flash sources, Edgerton's 1930's invention, provide ample energy (up to $0.5 \mathrm{~J}$ of photometric light output 


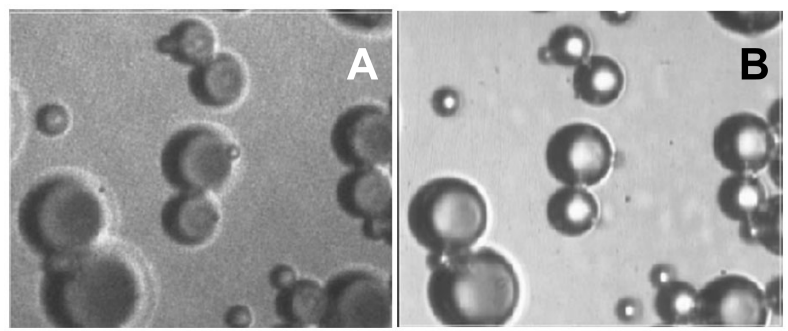

Fig. 16 Two ultra high-speed frames of the very same vibrating microbubbles. A: taken with an image-intensified system. The noise typically associated with the image intensifier is evident. B: taken with an ultra-sensitive unintensified CCD camera system.

per pulse), are well-controlled for syncing with the camera and can be as short as several nanoseconds. The pulse repetition frequency $(\mathrm{PRF})$ for high-intensity flashes is limited however, typically to $10 \mathrm{~Hz}$ or less. Increased flash rates are possible at the expense of decreased output energy. Lasers are also used for illumination. The laser output is spectrally intense and primarily of interest for monochromatic applications such as high-speed fluorescence imaging. Copper-vapor lasers offer a high repetition rate of $40 \mathrm{kHz}$. High-brightness light-emitting diodes (LEDs) become increasingly popular for illumination. LEDs are readily available in a variety of colors (matching the spectral response of the imaging device) and relatively inexpensive. Although high-brightness LEDs already seem very bright to the naked eye, the real advantage for high-speed imaging applications is their use in a pulsed operation for a very short duration (order $100 \mathrm{~ns}$ ) in a flash or burst mode. A dramatic increase of the intensity is accomplished by driving high currents through the diode; a pulse of up to $100 \mathrm{~A}$ can be driven through the LED without damaging it. The flash repetition rate depends on the LED housing's ability to dissipate the heat that is formed; see Sect. 3.5 for testing the sensitivity of high-speed imaging systems.

One way to compensate for the loss of the number of photons is to simply enlarge the pixel size. This is common practice in CMOS-based chips, which suffer from a higher noise-level than those equipped with a CCD chip. The typical size is $20 \mu \mathrm{m}$, but can be up to $66.5 \mu \mathrm{m}$ [67]. It should be noted that the gain in sensitivity by a larger pixel size is counterbalanced by the fact that while imaging at a microscopic level the required magnification decreases the illumination level by the same amount. In fact, in microfluidic high-speed imaging one would be better off with a smaller pixel size, as the requirements for magnification are then more relaxed and do not interfere with the limitations of optical diffraction.

Image intensifiers have been proposed to increase the sensitivity. First of all we should note that because of the milliseconds lifetime of the image on the traditional P-43 phosphor screen (see Sect. 2.3), image intensifiers are not well-suited for high-speed imaging applications. A P-46 and P-47 phosphor screen on the other hand has a considerable shorter phosphorescence of order microseconds and could potentially be used for high-speed 
imaging applications [68]. The use of these phosphors is hindered however by the presence of a slowly decaying 'tail' which leads to ghosting effects. More importantly we should note that the signal level is indeed increased with increasing gain on the image intensifier, but that this equally well holds for spurious noise that is amplified as well. The overall gain in sensitivity is therefore marginal, see Fig. 16. The noise is reflected in the image as a grainy overlay, typical for image-intensified images, and it therefore also reduces the resolution of the images. The main purpose of an image intensifier therefore remains to be to control the exposure time at nanoseconds timescale, as discussed previously in Sect. 2.3.

\subsubsection{Triggering}

Typically, triggering of a high-speed recording is essential, because we have a limited number of frames available for the recording. A proper positioning of the temporal region of interest is then required. If the number of recorded frames is not an issue and sufficient camera memory and storage space is available, triggering may still be useful, simply for convenience to instantly locate the high-speed event for further analysis. Triggering can be performed in several ways: manually using a trigger button, from a control device driving the system, and either mechanically, optically, or acoustically coupled to the electronics of the camera. Most digital high-speed cameras have a TTL trigger input. Optical triggering can be done with a laser-photodiode combination, with the object interrupting the laser beam. Acoustic triggering can be accomplished using a microphone or a hydrophone. Note that the speed of sound both in air and in water is relatively low and that a trigger delay will be experienced depending on the distance between sound source and the detector. In the special case of ultra high-speed imaging, especially those systems with a limited number of frames, such a delayed trigger will always trigger too late.

The above description is termed a post-triggered acquisition where the frames are collected after the trigger is received. The trigger signal in this case is referred to as the start trigger. Sometimes we are also interested in the conditions preceding the event under study, the trigger is only received after the event of interest. Or the event is too fast or too unexpected to trigger, e.g. in crack formation [69]. As an example, inkjet printing nozzle failures are extremely rare. On average, only once in a billion droplet ejections (only once during a 6 hour continuous run) a disturbance could occur, e.g. through air entrainment from a dust particle near the nozzle opening. In all these cases pre-triggered acquisition is essential. In a pre-triggered acquisition, the frames are already collected before the trigger signal is received. In such an application, the hardware initiates frame acquisition with a software function and stores the image data in a ring buffer in memory. The trigger signal in this case is referred to as the 

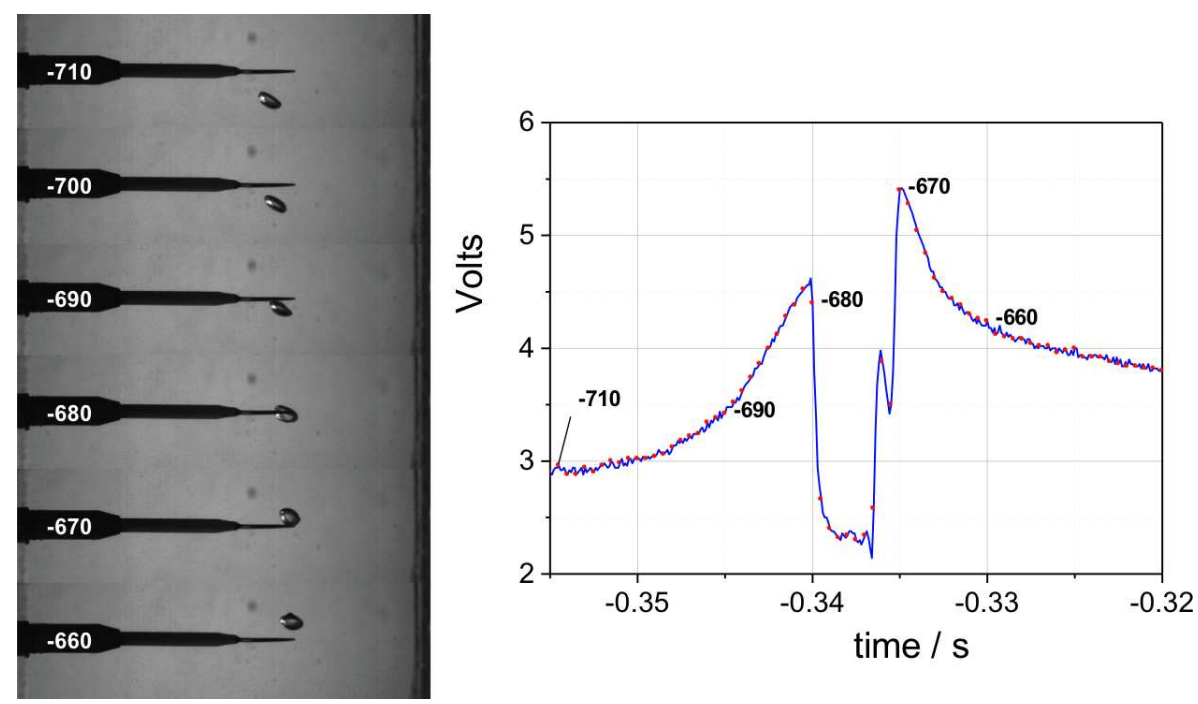

Fig. 17 Timing of a high-speed recording of a rising bubble interacting with a hot-wire probe. Each individual frame can be traced back in the hot-wire voltage recording (red dots) by simultaneous recording of each framing event directly from the high-speed camera system. The frame rate was 2,000 frames per second and the numbers indicate the frame numbers, at $500 \mu$ s intervals, before the actual trigger, using pre-triggering. (movie online).

reference trigger. The principle is quite similar to that of a digital storage oscilloscope, images are continuously stored in the buffer, and once the total record length is filled the oldest images are overwritten with the fresh images. Once the reference trigger is received, storage is halted, and the preset reference point for the trigger is set (with an arbitrary ratio of pre- and post-trigger frames), and images of the event before the actual triggering occurred are available.

The recording of Figure 3, for instance, is triggered by the acoustic signal emitted by the microbubble formed after pinch-off of the downward microjet. The falling droplet and the formation of the cavity were recorded before the trigger signal was received. And while this highly controlled experiment could also have been recorded in a post-triggered acquisition, e.g. by the falling droplet interrupting a laser-photodiode relay switch, all images of the implosion of the cavitation bubble of the snapping shrimp, Fig. 7, could only be taken with the pre-triggering option in function. Triggering occurred on the loud impulsive snap of the animal, admittedly after gentle tickling with a paintbrush, which only helped trigger the snap, not the timing of it. In modern digital high-speed cameras pre-triggered acquisition is standard and these systems can be slaved to the experiment. Some high-speed camera systems, e.g. rotating mirror cameras, are necessarily master to the experiment, and unless the high-speed event itself can be triggered pre-trigger images cannot be obtained. In some digital rotating mirror cameras pre-triggered acquisition can be implemented using smart electronic triggering.

The recorded frames need to be correlated in time. For this purpose a sync-out of the high-speed camera can be measured simultaneously with a trace related to the high-speed event, e.g. hydrophone recording. In some 
systems this is already implemented in the software. As an example we show recordings of the interaction of a rising bubble with a hot-wire probe [70], see Fig. 17. The purpose of the experiment was to obtain the energy spectra of a turbulent two-phase flow and to understand the two-way coupling of how rising bubbles modify the turbulence spectra and how the turbulent flow changes the bubble trajectories. This is a situation with a high gas fraction loading, which is really an experimental condition where optical techniques would fail miserably, hence the use of the hot-wire measurement technique. A typical trace is shown in Fig. 17, where we observe the drop in thermal conductivity of the hot-wire probe (for a duration of only $5 \mathrm{~ms}$ ) as soon as the wire protrudes the gas core of the bubble. Each red dot in the recording indicates a single frame of the high-speed movie, which are strictly correlated in time. The purpose of this particular high-speed imaging experiment of the hot-wire/bubble interaction was to uniquely link the measured hot-wire traces to whether the bubble was penetrated by the hotwire (and recovered, as shown in Fig. 17A), whether the bubble bounced off the hot-wire, or whether it splitted in multiple microbubbles as a result of the interaction with the heated wire [71]. A perfect correlation of the timing is therefore essential.

\subsubsection{Data}

The data stream in high-speed imaging can be considerable. We should differentiate the data stream during acquisition and the data stream after acquisition. The first can be on-chip and should accommodate very fast direct transfer to memory, as it directly relates to the corresponding refresh rate for imaging. The second data stream relates to storage and usually goes at reduced speed through USB, FireWire or Ethernet connection. Using flash SSD memory the number of images that can be stored in memory is extensive, where 32 GB is no exception. The total data stream, however, does not depend on the selected frame rate, as the image size is inversely proportional to the frame rate, see Fig. 14. We do record a lot more images at higher frame rates, but the images are also smaller. However, the high number of frames does impose latency in data transfer when transferring image data for off-line analysis.

Another important aspect that should be considered here is where to store the acquired data. For example, a 2-second recording taken at 5,000 fps will take nearly 7 minutes to review at a playback speed of 25 fps. More importantly, with an uncompressed image size of 1 Mpixel the total amount of data of that single event is 5 GB! A successful set of experiments in short time may result in a few hundred or-so recordings corresponding to 500 GB of data, which theoretically may take up to 2.5 hours to download over USB to an external hard drive with a 
transfer rate of $480 \mathrm{Mbps}$, but which typically proves to be very much longer in practice. Therefore it is important to develop pre-analysis software to quickly evaluate the recordings and distill the relevant observations; the true meaning of data reduction. Pre-triggering, as discussed in Sect. 3.3.2, greatly helps in these efforts. In practice, however, the data reduction and image post-processing and analysis consumes most of the work, and one may easily spend up to 2 months of work on analysis as a result of a single afternoon of experiments.

\subsubsection{The problem of throughput}

In Section 2.4 we have seen that there is a coupling between the temporal resolution and the spatial resolution. In high-speed imaging with commercial CCD or CMOS cameras there is yet another important coupling between the two. They are coupled in the sense that higher frame rates can only be achieved by reducing the amount of readout pixels, as a consequence of the upper limit of the total throughput rate of the digitizer; only a fixed amount of data can be pushed through the A/D convertor of the imaging chip, typically of order of several Gbits/s, or one billion pixels/sec. Strictly speaking the resolution remains the same if the magnification is unaltered, but in practice the tendency is to fill the field of view, which is then automatically associated with a loss in resolution once the frame rate is increased. A typical example is given in Fig. 18. Here two droplets are separated and a microscopic thread is being stretched until it snaps. Surface modes, which can be described by a superposition of Legendre polynomials, are formed and decay following they own mode eigenfrequencies, setting up a display of droplet shapes. The fast dynamics is captured by the high-speed camera at $100 \mathrm{kfps}$, and all the sequential shapes look beautiful, but in all honesty the image quality is not all that great. A single-shot photographic image well-timed with variable delays would do a much better job here.

Figure 14 shows an overview of how the total number of pixels in a frame recording depend on the frame rate for a set of popular high-speed imaging systems, taken from their datasheets. In the datasheet literature the full frames are referred to as frames (with the frame rate in frames per second (fps)), while the subdivided images are referred to as pictures (with the frame rate in pictures per second (pps)). A standard digital high-speed CMOS system records 1,000 by 1,000 pixels (=1 Mpixel) frames at a frame rate of 1,000 fps, but it quickly drops to about 100 by 100 pixels at a frame rate of $100 \mathrm{kfps}$. Frame rates, or to be precise, picture rates of 1 Mpps (pictures per second) can be obtained, albeit at the cost of a much reduced resolution, typically a strip of 8 pixels wide. Although this may be sufficient for the imaging of one-dimensional motion, it is not practical in most situations. 


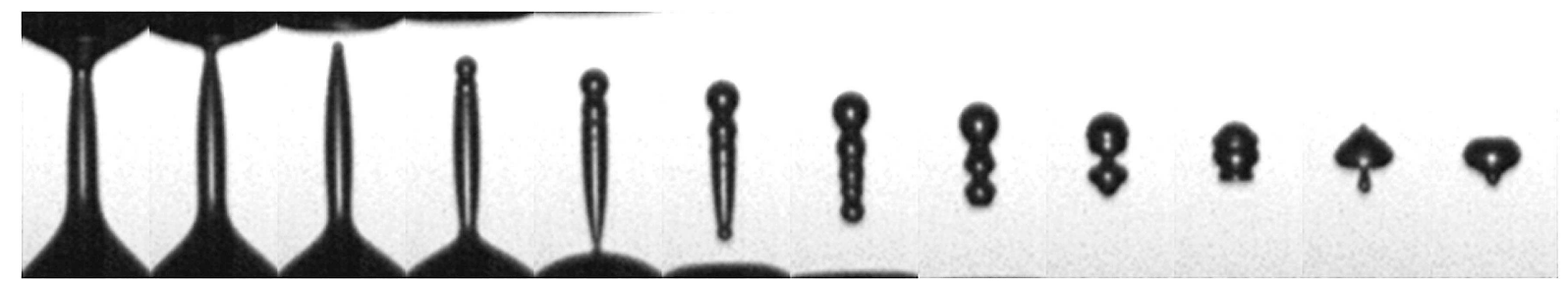

Fig. 18 A fine thread of liquid is stretched between two larger droplets. When the thread snaps, shape deformations are displayed. Frames taken at $100 \mathrm{kfps}$. This image sequence shows the resolution issue with limited pixel count of a high-speed CMOS cameras operated at high-frame rate (movie online).
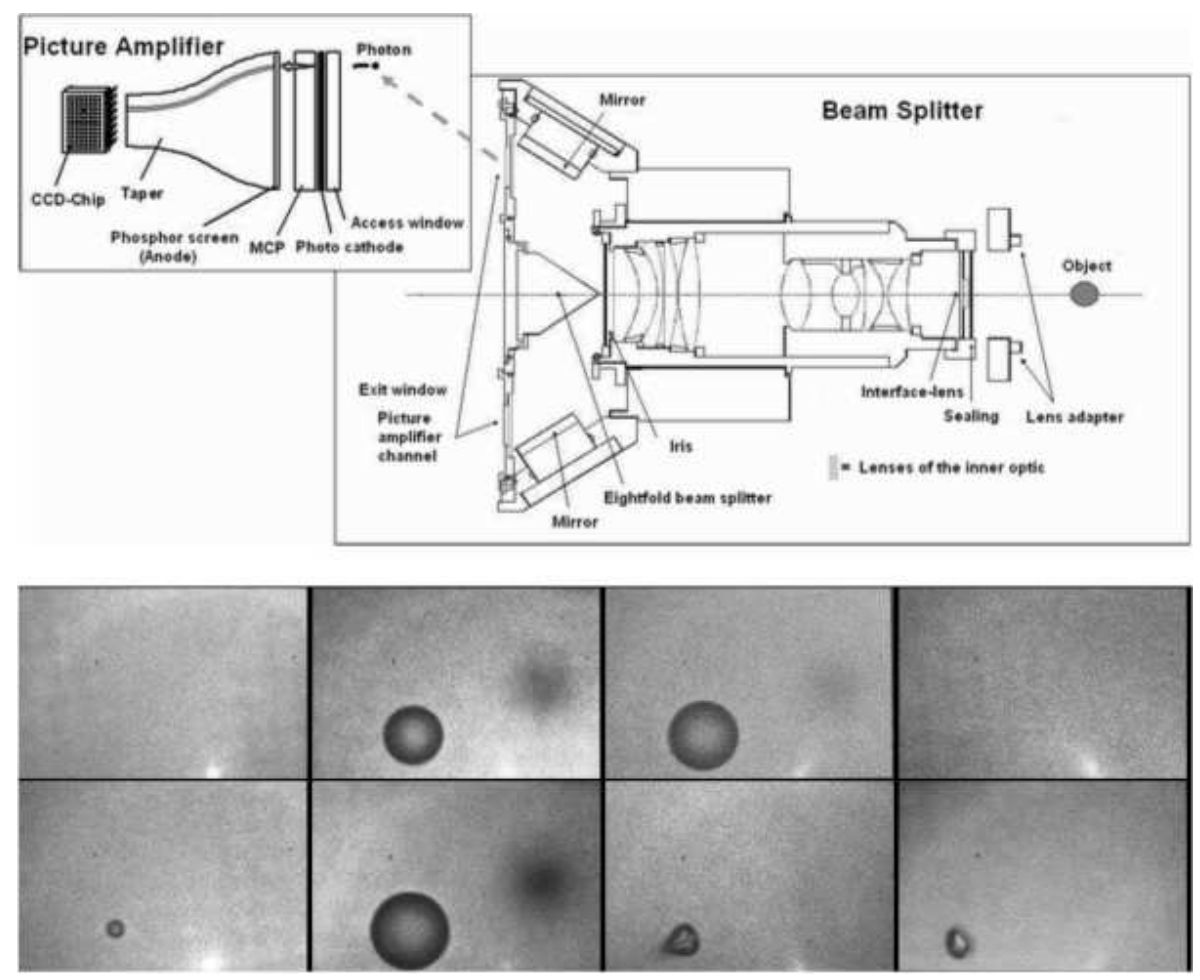

Fig. 19 Schematic of the Imacon 468 framing camera. The object image is relayed onto an eight-sided prism beam splitter and directed to eight image-intensified CCD camera with a gate time of 5-10 ns, which can be set independently, allowing ultra high-speed imaging at a frame rate of 100-200 Mfps. A typical recording of the eight images is shown below where a violently oscillating microbubble is captured shortly before an asymmetric collapse, where it displays microjet formation.

3.4 Ultra high-speed imaging systems beyond 1 Mfps

So what if we need to push the limits of current CCD and CMOS camera technology and perform high-speed imaging at exposure times shorter than 1 microsecond, and correspondingly at a higher sensitivity, and at frame rates higher than one million frames per second with a sufficient number of frames, typically one hundred or more. The simplest solution is to follow the classic example of Eadweard Muybridge and Berlyn Brixner by using a set of multiple cameras, as discussed in Section 1. The DLR German Aerospace Centre in Göttingen developed an eight-channel image-intensifed ultra high-speed framing camera for high-speed shockwave imaging, which was later commercialized through DRS Hadland as the Imacon 468 camera [46]. The Imacon 468 camera, 
see Fig. 19, utilizes a pyramidal beam splitter with an octagonal base that redirects the incoming image to 8 individual image-intensifed CCDs. Cordin Company and Specialized Imaging offer cameras with similar beam splitting configurations and 1 or 2 Mpixel chip size. LaVision's UltraSpeedStar camera uses an image splitter into four channels with color iCCDs, then split electronically the four RGGB channels to acquire 16 images at 1 Mfps. The image intensifiers can be gated as short as $5 \mathrm{~ns}$, leading to a frame rate as high as 200 Mfps for the Imacon 200, and very high gain levels can be applied that allow for sufficient sensitivity at such short exposure times. Each intensified CCD can be individually triggered with high accuracy and flexibility. The Imacon 200 can be equipped with P-47 phosphor backed image intensifiers, with rapid fall-off of the phosphorescence, which allows a second exposure per channel, giving $2 \times 8$ frames in total with 1-4 microseconds in between successive exposures to prevent residual image of the previous exposure, termed ghosting of the image. As discussed in Section 3.3.1, overall the image intensifiers seriously degrade the image quality. And as a result of the beam splitting, a high gain needs to be applied to the intensifiers, decreasing the dynamic range as a result of increased noise levels. On the other hand the system is unique in the 100-200 Mfps segment.

A rotating mirror camera does not suffer from the drawbacks of beam splitting or image intensifying and has a higher resolution, dynamic range, and frame count. A short exposure time is obtained by sweeping the image by rapid rotation of a turbine-driven mirror across a series of CCD sensors. The CCD sensors replace the traditional negative film used in the 1950's to record nuclear detonations. And while a nuclear detonation is bright enough to be captured with 3600 ISO number negative film, microfluidic applications with $100 \times$ magnification under a microscope certainly lack image brightness. Moreover CCD sensors are much more flexible, can be accurately timed and allow for multiple and repetitive exposures in short time. The Cordin 550 acquires 60 frames at 4 Mfps. The Brandaris camera, see Fig. 20, developed by University of Twente and Erasmus MC Rotterdam, is equipped with 128 CCD sensors, each of which can store 6 full frame images in its on-board RAM. The helium-driven turbine can rotate at 20,000 rps, completing a sweep across the image arc in just $5 \mu$ s providing an interframe time of 40 nanoseconds. The Brandaris camera can therefore acquire a total of 768 frames at a frame rate of $25 \mathrm{Mfps}$ in a set of $6 \times 128$ frames, and in segmented mode $12 \times 64$ frames or $24 \times 32$ frames [19,72] or any permutation of the above, provided that a $20 \mathrm{~ms}$ read-out time is required once all 128 channels are filled. A typical example of images taken with the Brandaris camera are included in Fig. 21. A third class of imaging systems utilizes in-situ storage of image data on the CCD chip itself. The Shimadzu HPV series camera [67] can record 103 frames in a single experiment at a maximum frame rate of 1 Mfps with a fixed resolution of $312 \times 260$ 


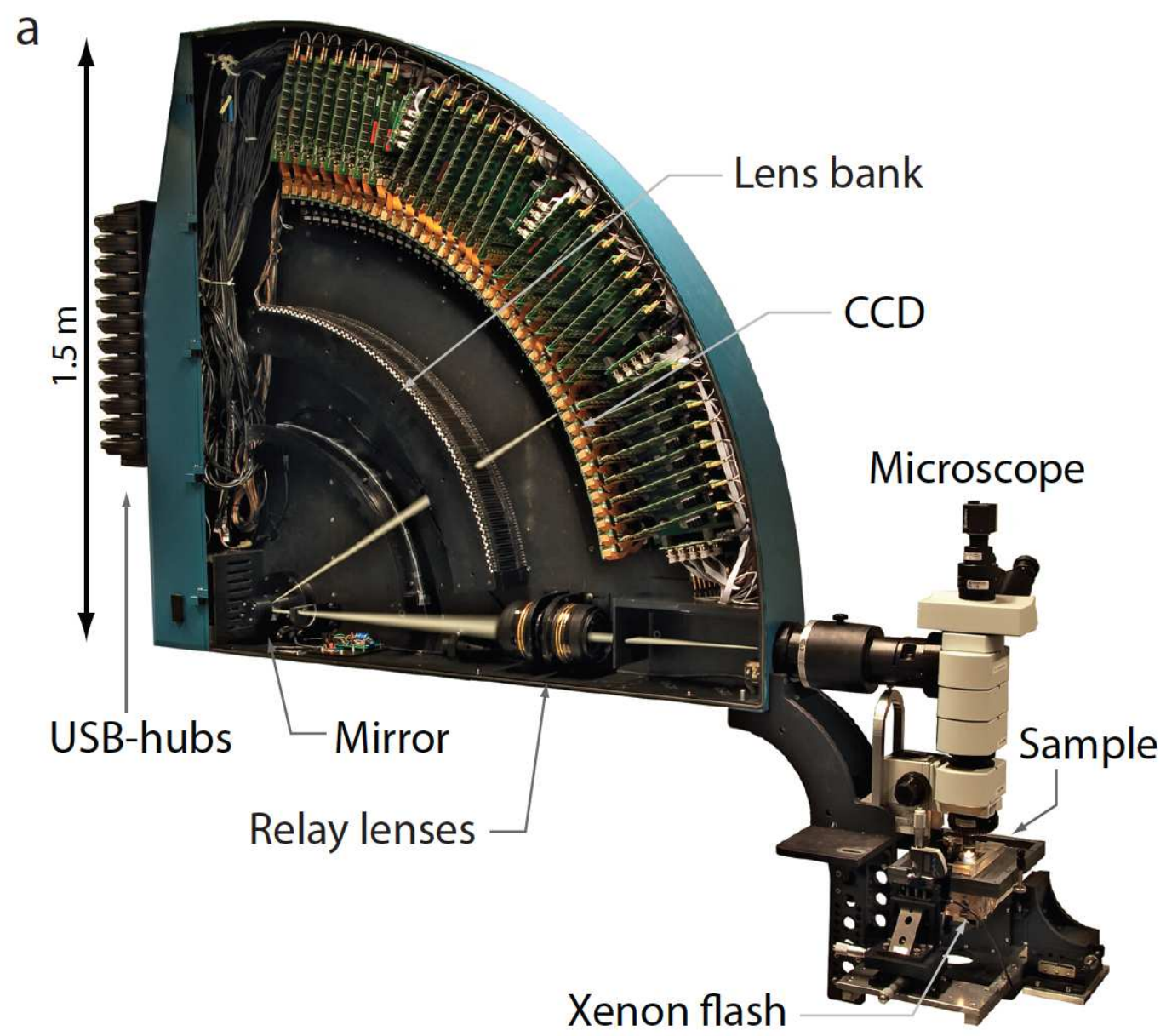

Fig. 20 The Brandaris-128 camera is a digital rotating mirror camera. It captures 128 frames in a single run at a frame rate of 25 million frames per second by sweeping the reflection of the incoming image across 128 highly sensitive CCD cameras. With the turbine running at $20,000 \mathrm{rps}$ such a sweep takes $5 \mu$ s leading to an interframe time of $40 \mathrm{~ns}[45,72]$. A total of 768 frames can be taken in sequential runs.

pixels. Its operating principle is similar to a two-frame storage CCD chip used in PIV imaging [74], only here it uses 100 slanted storage pixels per one photosensitive pixel, see Fig. 22. This gives the sensor a rather poor fill factor of $13 \%$, and limits its sensitivity, which is partly compensated by the large photosensitive pixel size of $66.5 \mu \mathrm{m}$. On the other hand the camera operates as a traditional electronic camera, with flexible timing options, including pre-triggering.

The future of ultra high-speed imaging is probably in this direction. Recent developments in CMOS technology include back-side illuminated (BSI) image sensors. Back-thinned BSI sensors have an increased quantum efficiency and through the absence of wiring on the back-side, which can be exclusively placed on the front-side of the chip, they have in principle a fill factor of $100 \%$. This development has ultimately led to the design of the ISIS-V16 sensor with a ten-fold increase in sensitivity and which can now acquire 117 frames at a frame rate of 16 Mfps [75]. Another development is the stacked image sensor. Stacking is a straight-forward extension of the BSI technology. 
Advanced circuit chips can be stacked on the front-side of the imaging chips. Commercial designs for consumer cameras are already marketed and this technology can be applied to develop various highly-functional images sensors for ultra high-speed imaging. As for the in-situ storage sensors recent solid-state technology became available using special CMOS capacitors as the in-situ memory. This system can operate at speeds exceeding 10 Mfps [76]. Combinations of all these new developments are expected to reach $100 \mathrm{Mfps}$ and beyond.

\subsection{High-speed imaging: testing and calibration}

A quantitative and objective determination of various physical parameters of the cameras (sensitivity, resolution, dynamic range, etc.) and light sources (brightness, spectral intensity, etc.) is welcomed. Testing conditions need to be constant and reproducible, and at the same time it has to be flexible enough to fit different sized sensor systems. That is why a black box design was developed that on one side was made to fit a light source, and on the other side, a sensor. The design is shown in side view in figure 24 . The pathway of the light is as follows: diverging light is emitted from the tip of a 7-mm optical fiber. The light from the optical fiber is attenuated by neutral density filters and a set of polarizers. The optical density of the filters ranges from 0.3 to 2.5 . One of the polarizers is fixed, while the other can be rotated which allows for the fine-tuning of the light intensity within the range of a single neutral density filter. The combination of the filters and polarizers results in a dynamic light range covering three orders of magnitude. The transmitted light falls onto a diffusing glass, which will evenly illuminate a resolution target (1951 USAF glass slide, Edmund Optics). The resolution target consists of 8 groups of each 6 elements of line pairs with a resolution varying from 1 to $228 \mathrm{lp} / \mathrm{mm}$. The green rectangle indicates the segment of the target that was used for the linearity, signal to noise ratio, sensitivity and dynamic

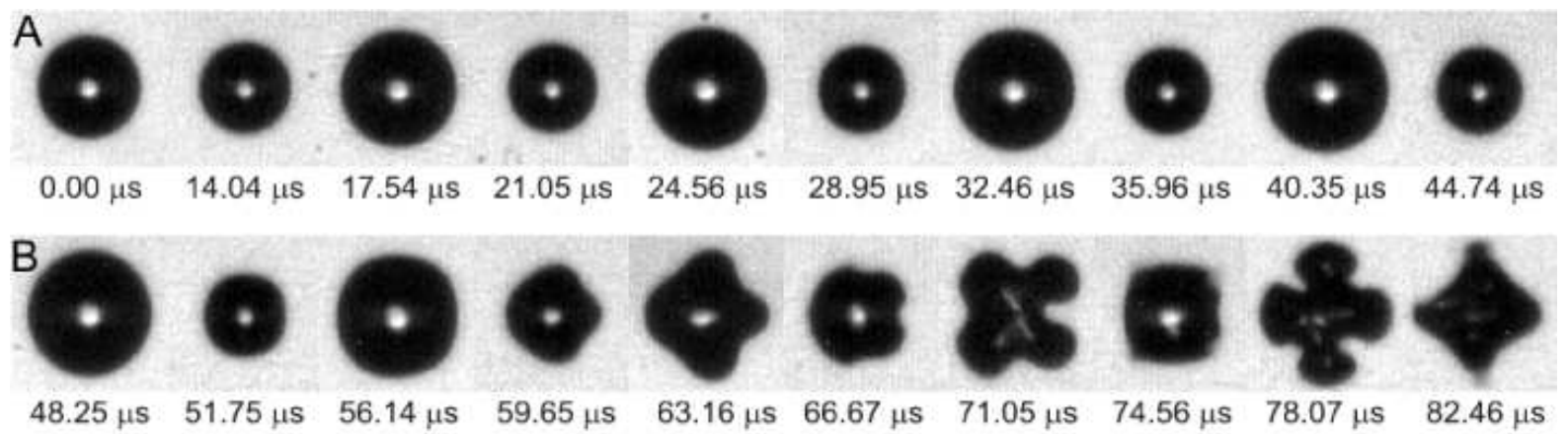

Fig. 21 Growth of a surface mode vibration for a bubble with a radius of $36 \mu \mathrm{m}$ as captured with the Brandaris high-speed camera. The bubble is driven by a 8-cycle ultrasound pulse at a frequency of $130 \mathrm{kHz}$ at an acoustic pressure of $120 \mathrm{kPa}$. First, the bubble oscillates in a purely volumetric radial mode (A), then after 4 cycles of ultrasound (B) the bubble becomes shape-unstable and a surface mode $n=4$ is formed [73] (movie online). 


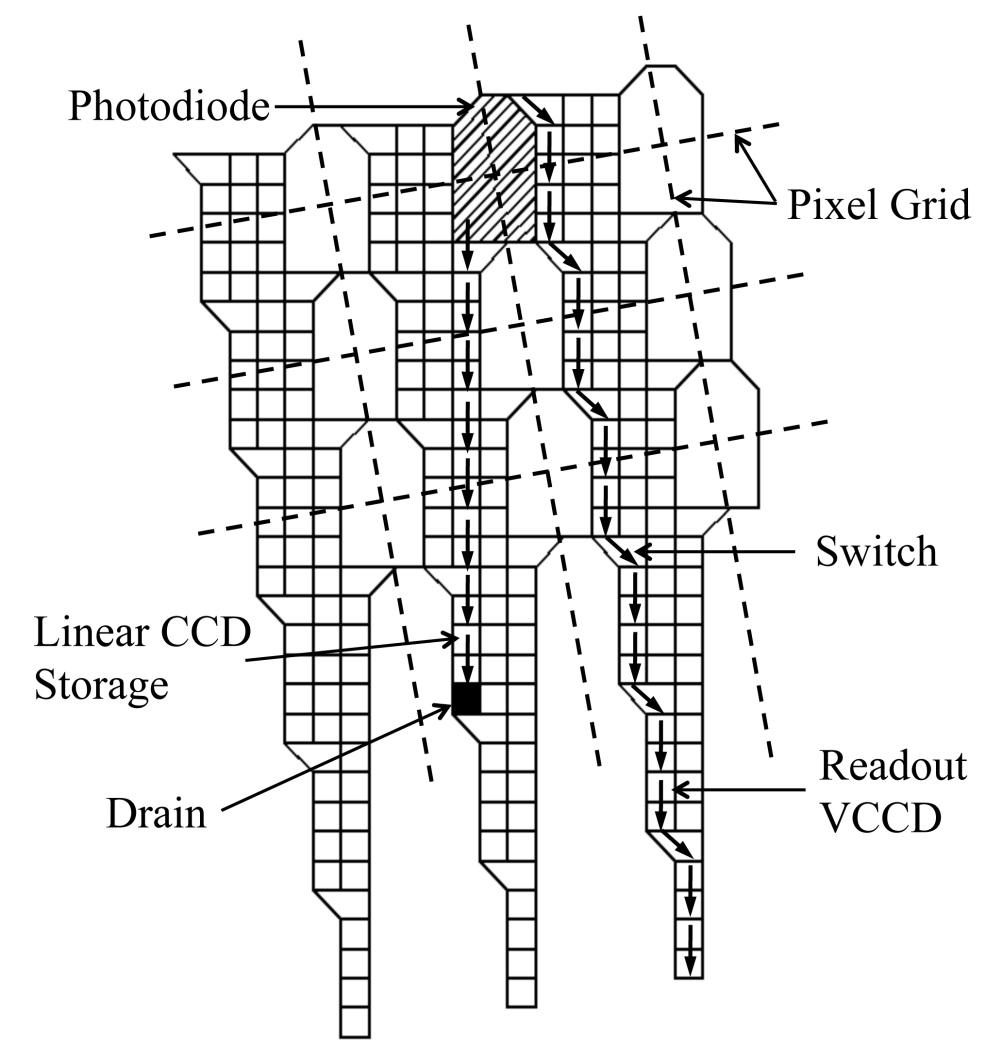

Fig. 22 Schematic of the ISIS chip [67] with slanted storage pixels design. The large photo-sensitive areas (grey) have a size of $66.5 \mu \mathrm{m}$. The charge is transfer to much smaller storage pixels $(\mathrm{N}=103)$ arranged in a clever design. Nonetheless the resulting fill factor of the sensor is only 14\%. A frame rate of 1 million frame per second can be achieved with this design and is currently used in the Shimadzu HPV-1 camera.

range measurements discussed below. The red squares show two groups of line pairs with different resolution.

A translation stage allows for centering and a goniometric stage for rotational alignment in the object plane.

An image of the resolution target is made by an objective lens (Carl Zeiss Makro-Planar T* $\mathrm{f} / 2.8-100 \mathrm{~mm}$ ) onto

the sensor. The lens has the ability to make a one-to-one conversion of the object onto the sensor. The image distortions and luminance differences within the field of view caused by the lens are negligible. The lens and sensor are mounted onto a translation stage in order to focus the object onto the sensor.

Now we define the test procedure for six elementary high-speed sensor properties that can be quantified with the test setup and which should aid at the selection of the optimum sensor:

\section{Dark current}

With a fully covered sensor, a single frame is recorded. The measured signal is the dark current that is caused by thermal energy in the CCD combined with the readout noise of the amplifier. The measurement will give two results: the background level and the noise. The noise is defined as the standard deviation of all pixel values whereas the background level is the mean. A high background signal will decrease the usable number 


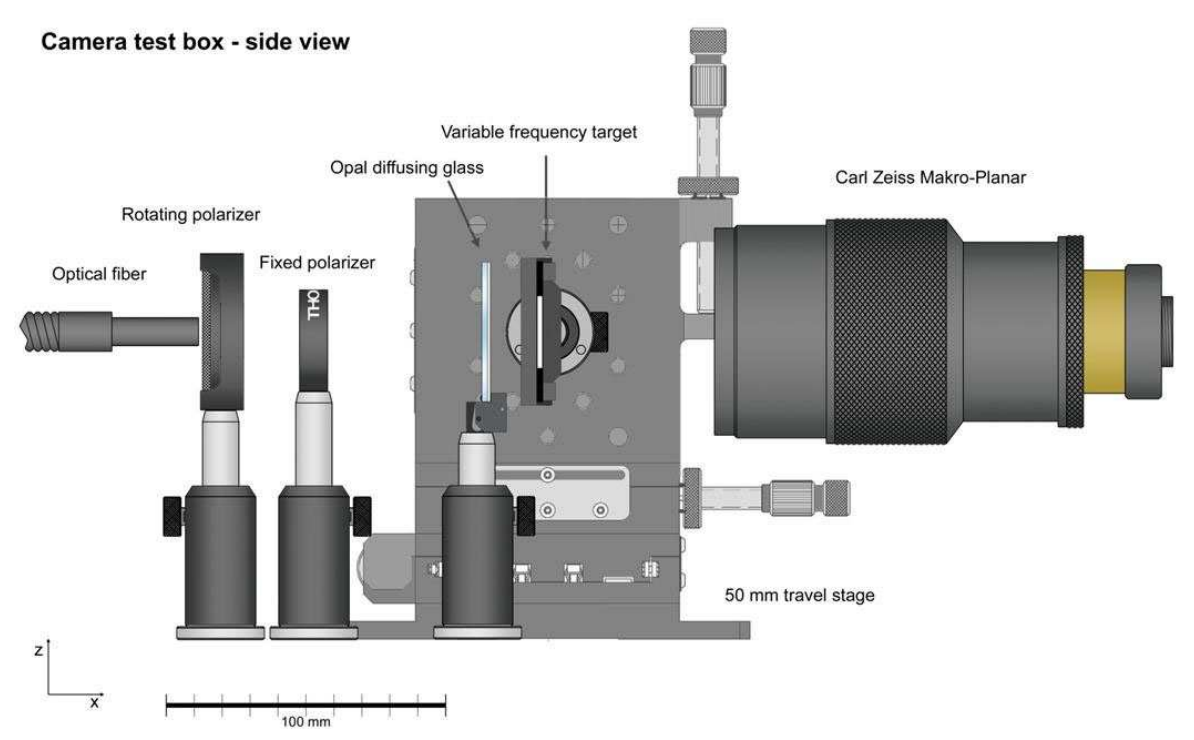

Fig. 23 Setup to calibrate imaging resolution and sensitivity. Various light sources can be coupled to the optical fiber to the left. A set of polarizers controls the light level. An imaging objective (Carl Zeiss Makro-Planar) focuses the image onto the sensor of the camera system under calibration.

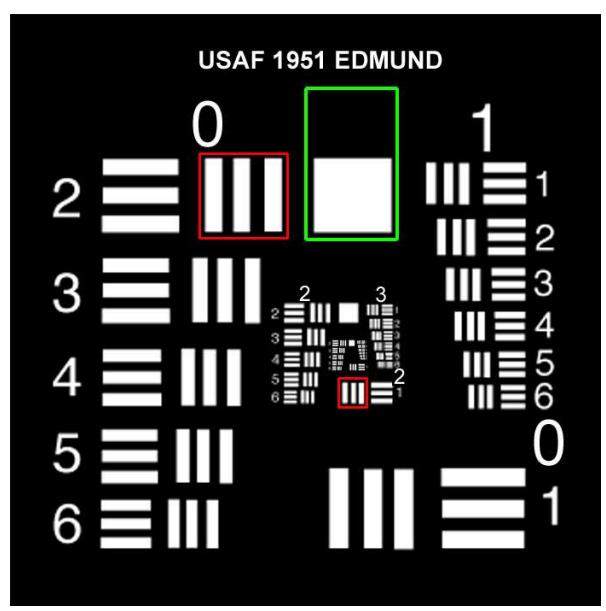

Fig. 24 The 1951 USAF glass target used for resolution testing. The test pattern is widely accepted to test the resolving power of optical imaging systems.

of gray levels and thereby reduce the dynamic range. When the noise is low, the background can easily be subtracted afterwards without image degradation. Therefore the standard deviation is more important than the mean value.

2. Linearity

Several images are taken at different light intensities to measure the linearity of the sensor. The sensor is said to be linear when the output signal level follows a line with constant slope as a function of the incident light intensity. Linearity is desirable in quantitative measurements where the image intensity can be correlated to a concentration of a dye or density of molecular species. 
3. Resolution

An important factor for the image quality is the resolution. This was measured by taking images of the different groups of the resolution target. For each element the modulation transfer function (MTF) was determined. The MTF is a measure of the contrast of an image at a particular resolution. It is also referred to as the spatial frequency response.

$$
M T F=\frac{(\text { signal }- \text { background })}{(\text { signal }+ \text { background })}
$$

4. Signal to noise ratio

This ratio relates the signal level to the standard deviation of the background. The higher the signal to noise ratio, the better a signal or object can be distinguished from its background. The ratio is measured for different light intensities.

5. Sensitivity

Sensitivity is the main property that needs to be evaluated for the different sensors. It is defined as the signal level with the background subtracted. Since light intensities in a high-speed imaging system are typically very low, the sensor should have a substantial signal level on top of the background level in order to distinguish between background and signal without considering the influence of noise. As with the linearity and signal to noise measurements, the signal is measured for different light intensities.

6. Dynamic range

It is not just the background that suffers from noise. Signal noise also influences picture quality and generally increases with increasing signal level. The standard deviation of the signal level mainly determines the number of gray-scales that an imaging system can distinguish. An evenly illuminated area of the sensor will give a distribution of pixel values which all correspond to the same gray level. The range of this particular gray level is given by the standard deviation of the pixel values. This determines the confidence interval of a pixel value i.e. the probability that a pixel value outside this interval does not belong to the same gray level. The total number of gray levels that fit within the complete range of the sensor is defined as the dynamic range.

With the full data set different sensor types can now be compared. To summarize, we want the dark current to be low, the sensor to be linear, its resolution high, its signal to noise ratio also high, the sensitivity high and the dynamic range large. This information together with several other considerations, such as size and price, in conjunction with operational complications, such as the need for cooling and possible electromagnetic 
interference, will then determine the optimum sensor for the applications, once it also fits the high-speed imaging considerations mentioned in the previous sections.

\section{Combined high-speed imaging techniques}

High-speed imaging and other experiments in fluids techniques are remarkable in their own entity, but combine them and you get something life-changing. Here we discuss various combinations of high-speed imaging with Schlieren and shadowgraphy, with particle tracking and particle imaging velocimetry, and we discuss high-speed fluorescence imaging.

4.1 High-speed shadowgraphy and Schlieren imaging

Both shadowgraphy and Schlieren imaging are well-known techniques to visualize density differences in a transparent medium [77]. Density differences cause a change in the light refraction index of the medium. Light traveling through the medium will experience a different refraction depending on the local density gradient of the medium. The Schlieren technique visualizes the spatial derivative of the refraction index, which can vary both through a change in density and temperature. Shadowgraphy is simpler to setup, however it relies on the 2nd derivative of the refractive index, hence it is difficult to quantify and serves primarily as a powerful visualization tool. Both techniques are broadly used in compressible flows, in which pressure fluctuations and therefore density differences are common. Shadowgraphy and Schlieren are traditionally used in combination with high-speed flash photography in windtunnel shockwave imaging [77]. One striking example is the photograph of the turbulent wake structure behind a supersonic riffle bullet by Ernst Mach [33]. The visualization of wake structures in liquid flows is more difficult. De Vries [78] was one of the first to report on high-speed imaging of wake visualizations behind rising spheres using a Schlieren setup. One important aspect is the non-intrusive nature of the Schlieren technique, as opposed to PIV [79] and dye injection [80]. The latter two are widely used and described in literature for the visualization of wake structures behind bodies[refs], both for solid spheres and bubbles, however the introduction of particles and fluorophores interacts with the slip boundary layers and interfaces of the rising objects, changing the wake vorticity and bubble shape. On the other hand, density differences in the water created by the motion of spheres and bubbles are too small for Schlieren to be detected. Therefore, a vertical temperature gradient is imposed on the quiescent water in the water tank. A heating lamp is positioned on top of the water tank, creating a constant vertical temperature gradient of $1.0 \mathrm{~K} / \mathrm{cm}$. The spheres and bubbles drag 
(a)

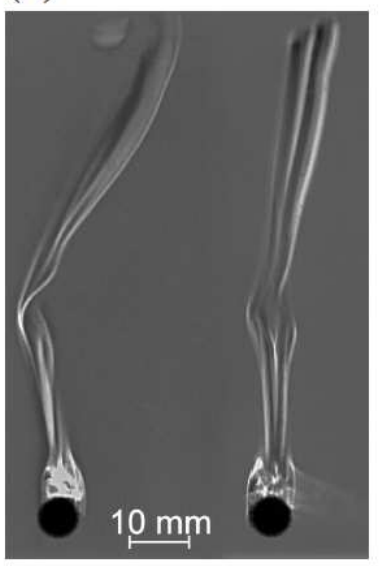

(b)

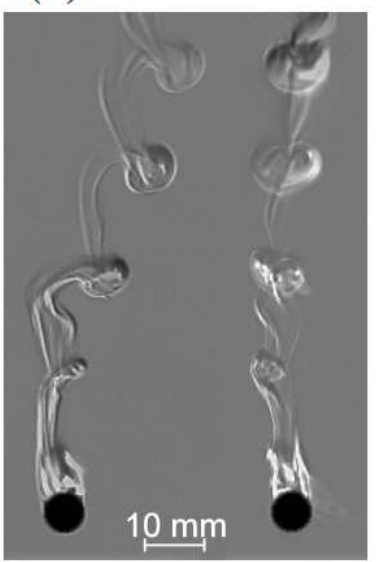

(c)

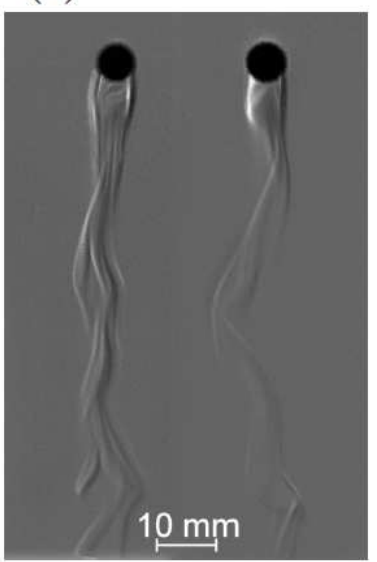

(d)

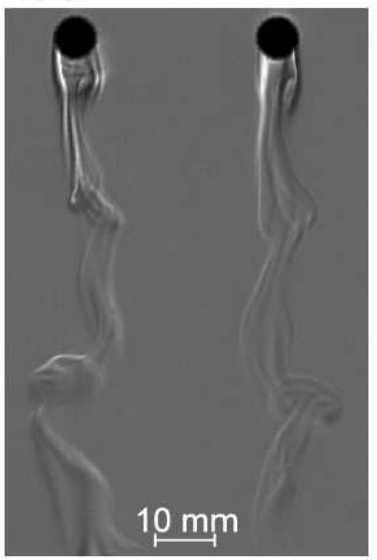

Fig. 25 High-speed Schlieren imaging displaying the wake structure behind falling and rising spheres [81]. Densities 4\% (A) and 5\% (B) higher than that of the surrounding liquid(movie online), and with densities that are approximately $4 \%$ (C) and $5 \%$ lower (D).

along water of different density, creating a local change in density, which can be visualized with the Schlieren technique. Of course, the introduction of high-speed imaging has also put a major challenge on the sensitivity issue of the setup.

Here we describe the construction of an orthogonal high-speed thermographic Schlieren imaging setup by transmitting the light originating from two LEDs $(0.12 \mathrm{~W})$ through a pinhole (diameter $1.5 \mathrm{~mm})$ with a diffusor to create a near-perfect light point-source. The two light bundles are collimated into two parallel light bundles with a diameter of $100 \mathrm{~mm}$. The two light bundles are passed perpendicular to each other through a water tank. After passage through the water tank the light bundles are focused onto a circular cutoff dot printed on a transparent plastic sheet (size range between 1-3 mm), and acting as the Schlieren knife edge. After the cut-off the light bundles were directed onto a high-speed camera. In the post-processing a background image is subtracted from all recorded images to provide a smooth background in each image.

Fig. 25 shows stereoscopic images of the wake structure behind falling spheres with densities approximately $4 \%$ (Fig. 25A) and 5\% (Fig. 25B) higher than that of the surrounding liquid, and, for comparison, that behind rising spheres with densities that are approximately 4\% (Fig. 25C) and 5\% (Fig. 25D) lower. In all these examples the sphere diameter is $6 \mathrm{~mm}$. The wakes of the falling spheres appear to have a more irregular structure, and the path followed by these spheres shows much larger deviations from a straight vertical line. These examples show the characteristic wakes for spheres following a zigzag path, namely that the two counterrotating threads in the wake cross at the centerline of the zigzag. The presence of these threads of opposite-signed streamwise vorticity implies that the sphere experiences a lift force. As a consequence of the periodic crossing of the threads this force 
is always directed towards the zigzag center-line, see for more details the work of van Wijngaarden [82] and that of Veldhuis et al. [81].

Shadowgraphy is a relatively straightforward technique for the visualization of shockwaves in fluids. It is being used for the display of shockwaves in aerodynamics and in wind tunnel supersonic flows. In medical therapy the accurate registration of the pressure waves, e.g. in shock wave lithotripsy (SWL) [83,84], shock wave therapy (SWT) [85], histotripsy [86], or high-intensity focused ultrasound (HIFU) [87] is of prime importance for quality assurance of therapeutic devices. During megasonic cleaning in the semiconductor industry shockwaves may harm the fragile nanofabricated structures on the substrate. Figure 26A shows the wavefront of such a shockwave. It originates from the collapse near the substrate surface of a bubble cluster (red arrow) formed in cylindrical micropits [88], which act as artificial nucleation sites for bubble cavitation. Here, an ultrashort flash photography method enabled shadowgraphy imaging of these waves. With the wave moving at the speed of sound $\left(c_{w}=1500 \mathrm{~m} / \mathrm{s}\right)$ the image was taken $220 \mathrm{~ns}$ after the bubbles collapsed. It indicates how fast these waves propagate at the small scale. If we were to visualize directly the propagation of such a shockwave, we could resort to stroboscopic laser or iLIF illumination [54] at fixed time delays (see Sect. 3.2) if the propagation of the pressure wave is reproducible. Fortunately, this typically is the case, except that the aftermath of the pressure wave passage, i.e. cavitation cloud formation, thermal ablation, etc, is more unpredictable and irreproducible. In this case we need to set aside the stop motion imaging and use high-speed imaging instead. If we were to image a 50-mm region of interest, then owing to equation 1 we need a non-trivial frame rate of 300 kfps. Earlier ultra high-speed framing studies on shock wave inception of cavitation were performed by Ohl [89] with the 8-frame Imacon 468. Figure 26B shows a high-speed visualization of a focused pressure wave generated with a piezoelectric device used for shock wave therapy [90]. The movie was recorded at a frame rate of $1 \mathrm{Mfps}$, and focusing of the shockwave is observed, as well as formation and collapse of single cavitation bubbles and the radial expansion of secondary shockwaves from these microbubbles.

4.2 High-speed holography and interferometry

While Schlieren and shadowgraphy are popular visualization techniques, they suffer from a lack of accuracy due to their first order and second order dependence, respectively, of the refractive index following the GladstoneDale relation $[77,91]$. Moreover Schlieren and shadowgraph methods do not offer enough resolution to measure quantitative details of shockwaves, even in a simple geometry [92]. Moreover, they cannot deal with the intrinsic 


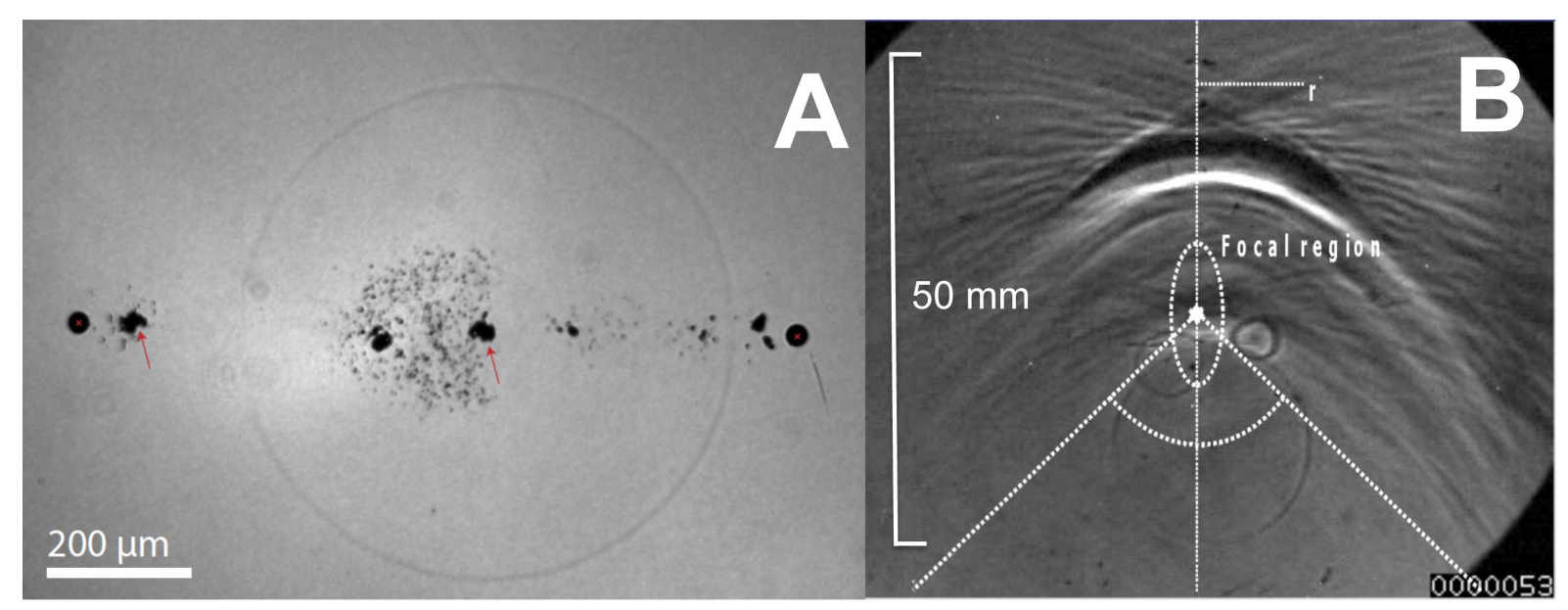

Fig. 26 A: High-speed shadowgraphy of a circular shockwave originating from a cavitation bubble collapse close to the center of micropit bubble clouds (indicated with the red arrows). The two micropits are marked with a red cross. Parts of another shock wave from an earlier event can also be distinguished, the origin of which is indicated with the left red arrow. B: Imaging of a focused shockwave using high-speed shadowgraphy. Bubbles are formed in the low-pressure region of the shockwave. Upon collapse they form spherical shockwaves which are seen in the lower part of B (movie online).

difficulty of measuring with a limited depth of field which is dictated by the geometrical optics, see the review by Kitzhofer et al. [93]. The solution is to revert to measurements in wave-optics using digital holographic imaging techniques, where the recorded phase image is an interference pattern from a superposition of a spherical scattering wave and a reference plane wave. The image contains information about the objects in the field of view with a nearly infinite depth of field [93,94].

Digital holography is used in a variety of fluids applications, including shocktubes, re-entry problems, and other compressible flows (see the excellent review on this topic by Kleine [92]), laser-induced processes such as ablation and optical breakdown in air or water. The latter for example suffers greatly from the formation of what appears to be chaotic multibubble clusters, shrouding part of the cavitation event and its growth. In order to capture the dynamics within the full context of space and time, holographic techniques have been employed making use of the three-dimensional character of image reconstruction of high-speed holograms, thereby obtaining views of the bubble distribution at different depths of field and at different viewing angles [95]. Similarly digital holography can be used for microdroplet characterization and for microparticle flow velocimetry, which explains its extensive use in PIV applications, see [93] and the references therein.

Interferometry is not been new to high-speed imaging, with the first interferometer built by Luwdig Mach in 1892 and independently by Zehnder in 1891. The Mach-Zender interferometer has regained popularity in today's high-speed imaging community with new emerging fast camera technology [96]. Compressible high-speed flows are visualized with a series of interferometry techniques, including speckle interferometry and differential interferometry [97]. The introduction of fast color high-speed cameras has facilitated digital color interferometry 
and color holography alike; both are popular techniques in high-speed flows such as in transonic wind tunnels and shocktubes. At the much smaller scale high-speed color interferometry has recently been used to measure the air layer thickness during drop impact on a solid surface at frame rates of up to $100 \mathrm{kfps}$ [98].

\subsection{High-speed Particle Tracking Velocimetry}

In the last few years 3D-Particle Tracking Velocimetry (PTV) has become a powerful measurement technique in fluid mechanics. The rapid development of high-speed imaging has enabled a successful implementation of the technique in studies on turbulent motion of particles [99-101], in thermal convection flows [102,103], and bubbly flows [104-106]. For the latter, the measured 3D spatial position of particles and bubbles and their associated time trajectories allow for a Lagrangian description which is the natural approach for the study of this type of transport mechanisms. Fig. 27 sketches the positions of four high-speed cameras (Photron 1024-PCI) which were used to uniquely determine the positions of seeding particles and bubbles in the flow. The four cameras were viewing from one side and focused in the central region of a water channel filled with bubbles injected from 500- $\mu$ m diameter needles mounted on three capillary islands. Camera objectives with a 50-mm focal length allowed for a depth of field of $6 \mathrm{~cm}$. The image sampling frequency was 1,000 $\mathrm{Hz}$ with a camera resolution of $1024 \times 1024$ pixels. The cameras were triggered externally in order to achieve a fully synchronized acquisition. We used the PTV software developed at ETH Swiss Federal Institute of Technology for camera calibration and tracking algorithms. For a detailed description of this technique we refer to the work of Hoyer et al. [107] and references therein. A 3D solid target was used for 3D spatial calibration. Different bubble concentrations were achieved by varying the air flow through the capillary islands. We performed experiments with dilute bubbly flows with typical void fractions $\alpha$ in the range $0.28 \%-0.74 \%$. A mono-dispersed bubbly swarm with mean bubble diameter of $4-5 \mathrm{~mm}$ was studied. Typical Reynolds numbers $R e$ are of the order $10^{3}$, the Weber number $W e$ is in the range 2-3 (implying deformable bubbles) and Eotvos number Eo around 3-4.

Bubbles were detected within a volume of $16 \times 16 \times 6 \mathrm{~cm}^{3}$ with an accuracy of $400 \mu \mathrm{m}$. To illuminate the measuring volume homogeneous back-light and a diffusive plate were used in order to get the bubbles contour imaged as a dark shadow. The image sequence was binarized after subtracting a sequence-averaged background; then these images were used along with the PTV software to get the $3 \mathrm{D}$ positions of the bubbles. We acquired 6400 images per camera corresponding to $6.4 \mathrm{~s}$ of measurement (6.7 GB of image files). For the most dilute case ( $\alpha=0.28 \%)$ around $N_{b} \approx 190$ individual bubbles were detected in each image. This quantity dropped to nearly 


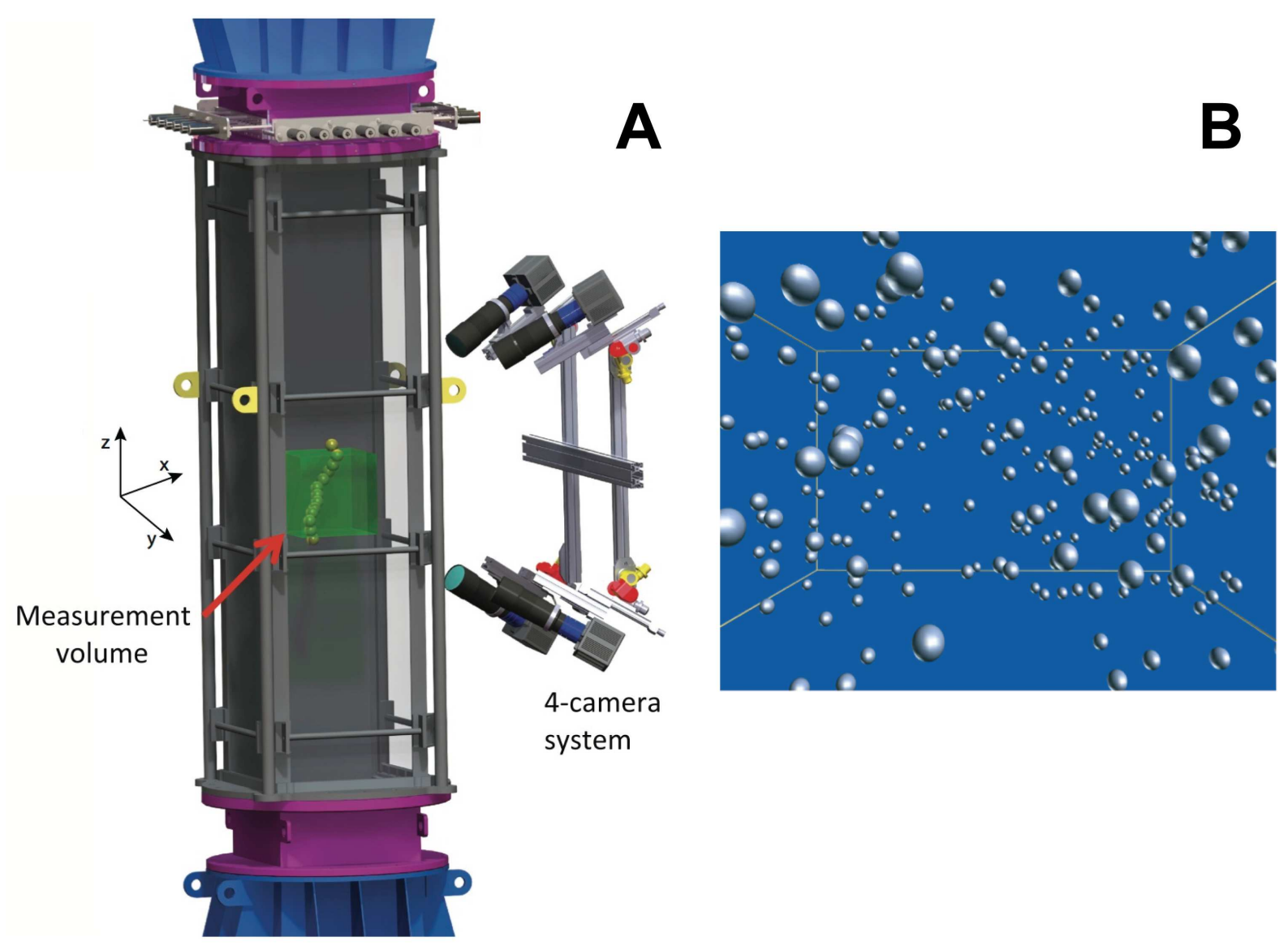

Fig. 27 3D-Particle tracking velocimetry utilizes four high-speed cameras to uniquely track the fluid motion and the bubble trajectories simultaneously. A: Schematic of the arrangement of the four high-speed cameras with respect to the measurement section. B: Snapshot of bubble positions obtained from a high-speed 3D-PTV recording set (movie online).

100 for the most concentrated cases $(\alpha=0.65 \%$ and $\alpha=0.74 \%)$ as for the higher void fraction, various bubbles cannot be identified as single objects. For even higher gas fractions hot-wire anemometry (CTA) was employed, see section 3.3.2. If a bubble could be tracked for at least 3 consecutive time steps, we call it a linked bubble, and the bubble trajectories were coupled to the 3D PTV data for further detailed analysis.

\subsection{High-speed Particle Imaging Velocimetry}

Combined with Particle Imaging Velocimetry (PIV), high-speed imaging is a powerful tool for quantitative analysis of the flow, see e.g. the recent work on flow behind blast waves by Murphy and AdrianciteMurphy2010. As an example we describe a micro-Particle Imaging Velocimetry ( $\mu$ PIV) setup suitable for the analysis of the flow in microscopic channels in a single plane. The release of neutrally buoyant tracer particles within the flow is a procedure that aids enhanced visualization of the flow pattern. Such particles are expected to follow the path of the fluid with accuracy $[108,109]$. A conventional PIV setup makes use of a laser sheet to illuminate 
(fluorescent) tracer particles to visualize and analyze the flow. In the present $\mu$ PIV setup, an objective with a small depth of focus and a continuous light source were used instead of a laser sheet. The main advantage of this setup was that the recording speed was not restricted by the amount of light emitted from the fluorescent particles. Therefore, recordings could be made both at high frame rate and for a prolonged time. A prolonged recording time allowed for ensemble averaging, which reduces the noise in the individual PIV recordings [109]. Here we show results from dentistry, notably the flow visualization of the irrigation in endodontic channels. Irrigation is a common procedure during a root canal treatment. An antimicrobial solution, typically sodium hypochlorite $(\mathrm{NaOCl}$, bleach), is flushed though an open-ended, or a side-vented, needle into the confinement of the root canal, leading to an axisymmetric, or, non-axisymmetric jet, respectively. The aim of this study was to quantify the amount of shear exerted on the root canal wall in various confinements, and to understand the irrigant refreshment mechanisms, in a range of Reynolds and Damköhler numbers, and to compare with CFD modeling [110].

The typical flow velocity $u$ inside the root canal is $1 \mathrm{~m} / \mathrm{s}$ and its typical dimension $l$ is $100 \mu \mathrm{m}$. Thus, following equation 1 , the frame rate required to capture the dynamic flow behavior of needle irrigation techniques must be higher than $100 \mathrm{kfps}$, depending on the applied magnification. Here we use a high-speed camera capable of recording 100 frames at a maximum frame rate of 1 Mfps (HPV-1, Shimadzu). The camera was attached to a microscope (BX-FM, Olympus) for $1.25 \times$ to $20 \times$ magnification. Illumination was provided for bright field imaging by a high intensity continuous metal-halide cold-light source. The flow through the needles was driven by a syringe pump at flow rates in the range 1 to $10 \mathrm{~mL} / \mathrm{min}$. The corresponding Reynolds numbers span two orders of magnitude, $10^{2}$ to $10^{3}$. The flow was allowed to start up for three seconds before the start of a measurement. Monodisperse hollow glass spheres with a mean diameter of $10 \mu \mathrm{m}$ and a mean density of $1.1 \times 10^{3} \mathrm{~kg} / \mathrm{m}^{3}$ were added to distilled water as tracer particles for $\mu \mathrm{PIV}$. The tracer particles fulfilled the requirements for $\mu \mathrm{PIV}$ imaging: they are neutrally buoyant, and they have a diameter much smaller than the typical length scale. For this system the Stokes number $S t=$ (particle response time)/(typical flow time) $<1$, even for the highest velocities.

Traditional PIV techniques rely on two consecutive photos illuminated with a laser sheet and light-scattering particles to visualize a thin plane in the volume of interest. However, in $\mu \mathrm{PIV}$ such a laser sheet would have a thickness of the same order as the volume of interest. Therefore here the total volume is illuminated and the depth of focus of the microscope objective is used to image only a thin image plane. The depth of measurement $d z$ of 

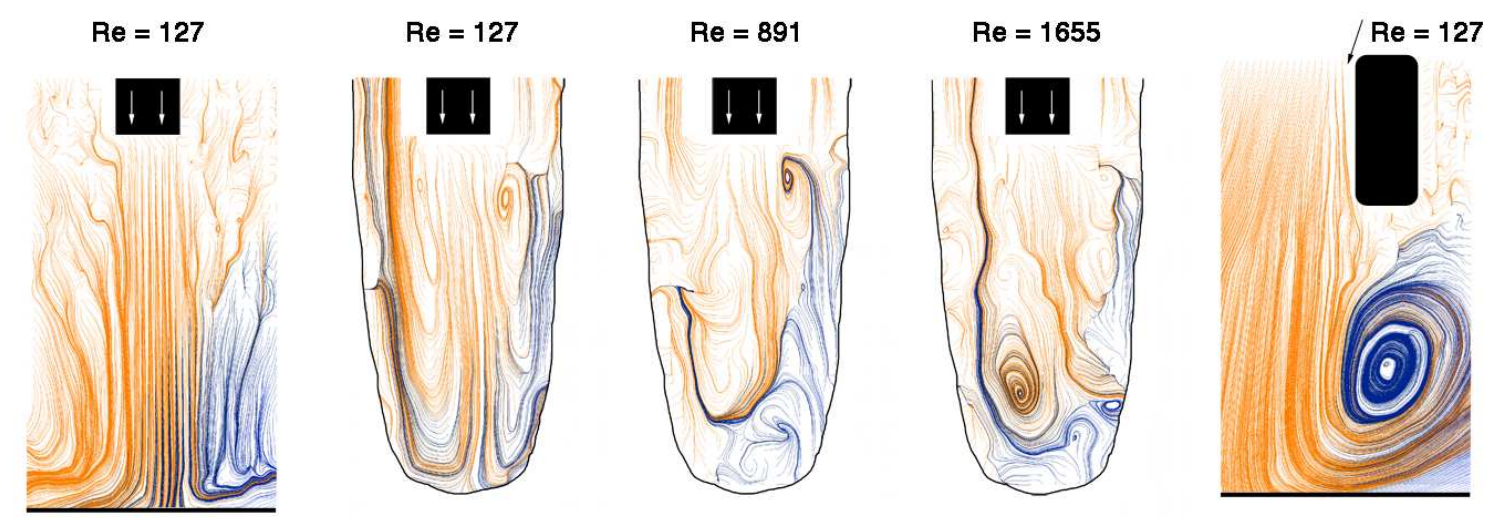

Fig. 28 Streamlines obtained from high-speed PIV imaging using different needle types for endodontic irrigation and removal of the biofilm in the root canal for various Reynolds numbers of the flow. Left-most image shows streamlines impinging on a flat plate. Right-most image shows the flow from a side-vented needle, which reduces the pressure in the apical part of the root canal, and which is an important safety precaution to prevent penetration of the bleach irrigant into the periapical tissue surrounding the tooth, where it can result in tissue damage and patient discomfort.

a $\mu \mathrm{PIV}$ system is then defined as twice the distance from the focal plane where the particles become sufficiently unfocused that they do not contribute to the velocity field [111]. For an objective with NA=0.25 imaging 10 $\mu \mathrm{m}$ particles scattering broadband light through air, the depth of measurement is approximately $100 \mu \mathrm{m}$, which indicates that the captured particle movement was limited to a plane with thickness of approximately $1 / 5$ of the root canal diameter and 10 times the particle diameter. The flow direction and velocity magnitude is obtained from the recordings of particle motion by calculating the cross-correlation between (parts of) two consecutive frames. Sub-pixel accuracy can be obtained by fitting a Gaussian curve to the $2 \mathrm{D}$ cross-correlation. The accuracy can be increased further using image processing techniques and data validation strategies. From the obtained velocity components $u$ and $v$, the vorticity $\omega=\partial v / \partial x-\partial u / \partial y$ can be obtained. This equation can be discretized and applied explicitly to the PIV velocity data.

Each recording consisted of 100 frames, which allowed for ensemble averaging [109]. A cross-correlation is made for each pair of frames, and these cross-correlations are added. This results in a higher peak in the final cross-correlations, which is equivalent to a higher signal-to-noise ratio. However, ensemble averaging reduces the temporal resolution and is therefore only suitable for steady flows. Non-steadiness of the flow was not observed within those 100 frames; therefore it was assumed that ensemble averaging did not cause a loss of temporal detail. The streamlines representing the measured flow patterns for the two types of jets are shown in Fig. 28. The global features of the measured velocity patterns match those predicted by the CFD model [112], showing two distinct velocity patterns: a straight, divergent jet in the case of the axisymmetric jet and a cascade of eddies declining in 
strength for the non-axisymmetric jet. The measured velocities were of the same order of magnitude as calculated by theory and the CFD model, although the highest velocities were not always observed in experiment.

The accuracy of the $\mu \mathrm{PIV}$ technique depends on many factors, both in the measurement equipment and in the analysis. The error in the analysis can be estimated by taking an image representative for the experiments that need to be analyzed, and displacing this image over a certain number of pixels. The PIV analysis should result in velocities equal to the displacements applied to the image. The script used in [112] showed that the applied displacements could indeed be recovered, within an error of approximately $5 \%$, which can be attributed to noise. This error analysis also pointed out that the result of the PIV analysis is very sensitive to the amount of details in the image, as low-contrast areas in the image will result in a zero velocity. An extension to threedimensional velocity measurements would be very useful considering the essentially three-dimensional flow inside a typical microfluidic channels. 3D-PIV in microfluidics is not easy to setup, but may be possible by estimating the out-of-focus component of consecutive 2D slices $[113,114]$.

\subsection{High-speed fluorescence imaging}

Laser-induced fluorescence (LIF) is frequently used in fluids research, such as the in flow visualization of turbulent mixing [117-119] and combustion processes [120-122], in (micro) particle image velocimetry $(\mu \mathrm{PIV})[123,74]$, and in molecular tagging [124-127]. Molecular fluorescence imaging uses pulsed dye laser tuned for resonant spectroscopic excitation, while for the measurement of velocity fields pulsed Nd:YAG lasers provide the nonspecific fluorescence excitation of micron-sized or nanometer-sized tracer particles. The typical pulse repetition frequency $(\mathrm{PRF})$ of these lasers is on the order of $10 \mathrm{~Hz}$ and a separate laser cavity would be required for each individual frame to be able to perform LIF at high frame rates. To sketch the complexity of such a high-speed fluorescence experiment we describe the joint effort of the Combustion Research Centers of Lund and Delft Institutes of Technology [115] to visualize time-resolved planar LIF in the Delft piloted turbulent jet diffusion flame [116] at a timescale smaller than $100 \mu \mathrm{s}$, which corresponded to the smallest turbulent Kolmogorov length scales of the non-premixed flame. The laser system consisted of a Nd:YAG laser cluster with 8 laser heads that could be individually triggered. Laser-induced fluorescence of $\mathrm{OH}$ radicals excited at a wavelength of $283 \mathrm{~nm}$ were imaged at a fluorescence wavelength of $309 \mathrm{~nm}$. The fluorescence was imaged onto a custom modified 8-channel ultra high-speed camera (Imacon 468, DRS Hadland, UK) [46], which consisted of 8 UV-sensitive intensified CCDs and which could record the 8 individual frames at a minimum interframe time of $10 \mathrm{~ns}$, corresponding to 

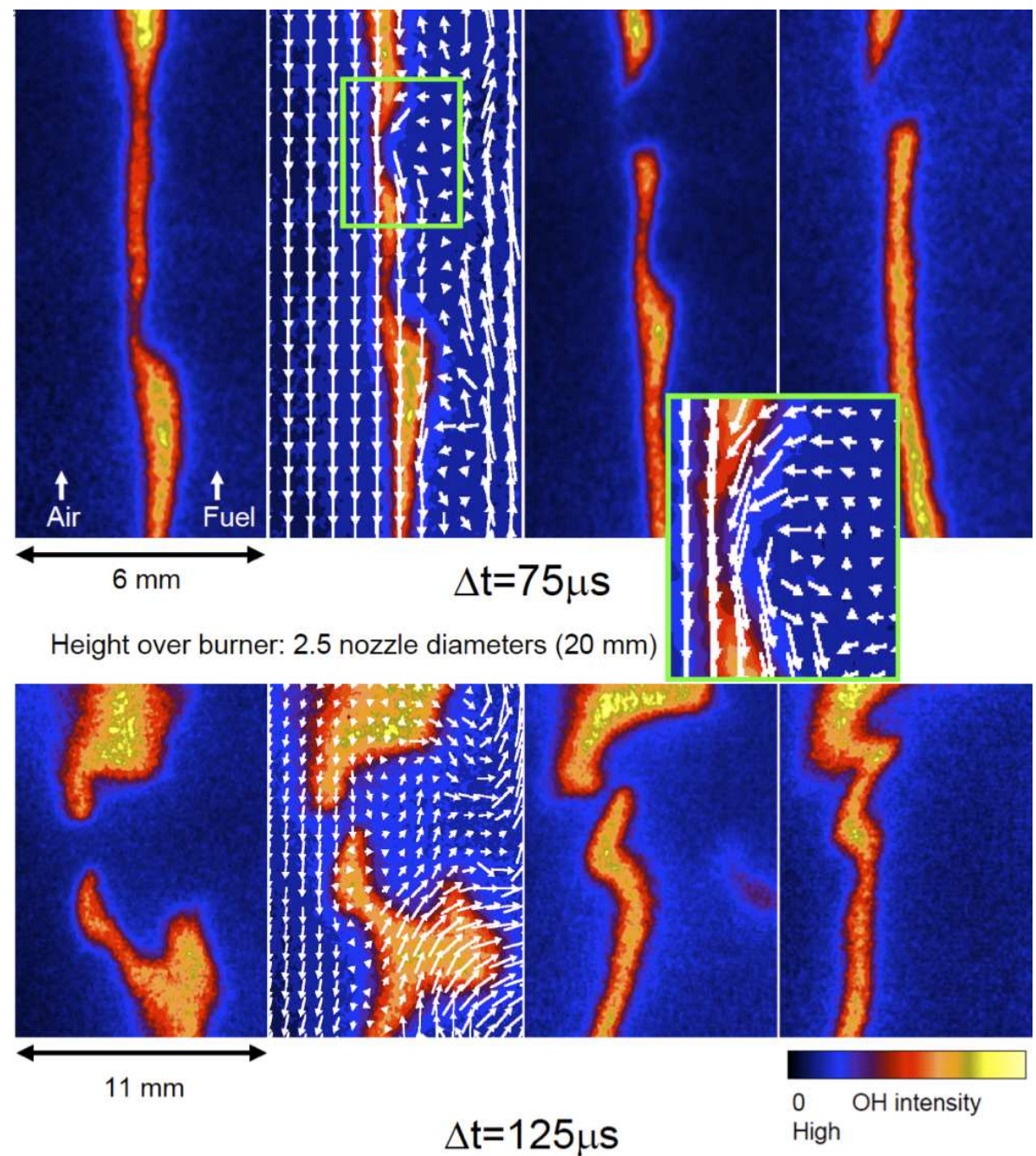

Fig. 29 High-speed imaging in turbulent combustion [115]. A laser cluster of $8 \mathrm{Nd}$ :YAG lasers and an eight-frame ultra high-speed camera resolves extinction and re-ignition of a turbulent jet diffusion flame [116] combined with PIV imaging.

100 Mfps. The fluorescence images were used for flame front tracking and the interframe time was set to $75-100$ $\mu$ s based on the turbulent timescales of the flame, which had a Reynolds number of 9,600. Fig. 29 shows typical results of the time-resolved planar LIF measurement in the turbulent diffusion flame. It shows the turbulencechemistry interactions, notably the local extinction of the flame and the timescales of its re-ignition. These flame dynamics measurements were also combined with PIV measurements, using two of the laser heads at $532 \mathrm{~nm}$ and using $\mathrm{TiO}_{2}$ as tracer particles $(\mathrm{d}=1 \mu \mathrm{m})$. These measurements showed the underlying mechanisms of extinction through the penetration of fuel vortices through the stretched flame front structure.

A series of 8 frames is limited in view of more extended studies involving transients or harmonic responses, where 100 or so time-resolved recordings would be required. From a cost-perspective, pulsed lasers are then considered unsuitable, especially when taking into account the required power supplies and cooling equipment. A CW laser does not reach the high peak intensity levels of a pulsed laser, however it can utilize the full exposure 
time to excite the fluorescent molecules multiple times. Moreover, diode-pumped solid-state lasers come in a large variety of wavelengths, they have a small form factor, they do not require extensive cooling, and they are much more affordable than a pulsed laser system. Here we discuss the combination of a 5 Watt 532-nm DPSS CW laser with the Brandaris-128 high-speed imaging facility [45], a system now capable of recording ultra high-speed fluorescence at up to 25 million frames per second. To facilitate a fast rise time and short pulses, the laser was gated by an acousto-optic modulator (AOM). The light transmitting efficiency of the AOM is $92 \%$, resulting in a maximum power of $4.8 \mathrm{~W}$ for a gate time of $5 \mu \mathrm{s}$, sufficiently long to illuminate all 128 channels of the camera with a 40 nanoseconds frame interval. At the same time the AOM avoids overexposure and heating of the (biological) sample.

The modular design of the microscope allowed for coupling of the laser light into the optical path of the microscope via a dichroic mirror. A $60 \times$ water-immersion objective with a numerical aperture of 0.9 , typically used for microbubble experiments, produces a spot with a full width at half maximum (FWHM) of $1.0 \mu \mathrm{m}$ and a a maximum laser intensity of $72 \mathrm{MW} / \mathrm{cm}^{2}$. The spot diameter can be increased by making the laser beam divergent before it enters the objective. When recording in bright field with the fluorescence filters in place, $42 \%$ of the Xenon flash illumination is filtered out by the dichroic mirror and a 532-nm laser notch filter, maintaining sufficient light levels for bright field imaging. With the fluorescence module in place, the Brandaris- 128 configuration gives rise to three illumination options: bright field, fluorescence, and the combination of both illumination techniques. For each individual recording within one experiment, the illumination method can be predefined. In the case of 6 full-frame recordings for instance, the first 3 can be captured in bright field and the final 3 in fluorescence. Other options include alternating bright field and fluorescence or even 12 combined recordings of which each recording is split into two virtual segments: the first 64 frames are recorded in bright field and the second 64 frames are recorded in fluorescence.

Coated microbubbles have been extensively studied and characterized in their role as ultrasound contrast agents, quantifying the perfusion of organs, for molecular imaging detecting molecular signatures of diseases with single bubbles in the body, and for drug delivery and therapy. A major drawback of bright-field imaging of microbubbles is the poor contrast of the bubble interface due to a complex interplay of refraction and scattering of the light transmitted in back-illumination. In addition, bright field imaging lacks the ability to distinguish between liquid media with similar refractive index, which is crucial for the visualization of drug release. Thus, ultra high-speed fluorescence imaging is a very promising experimental tool for the study of the above medical 

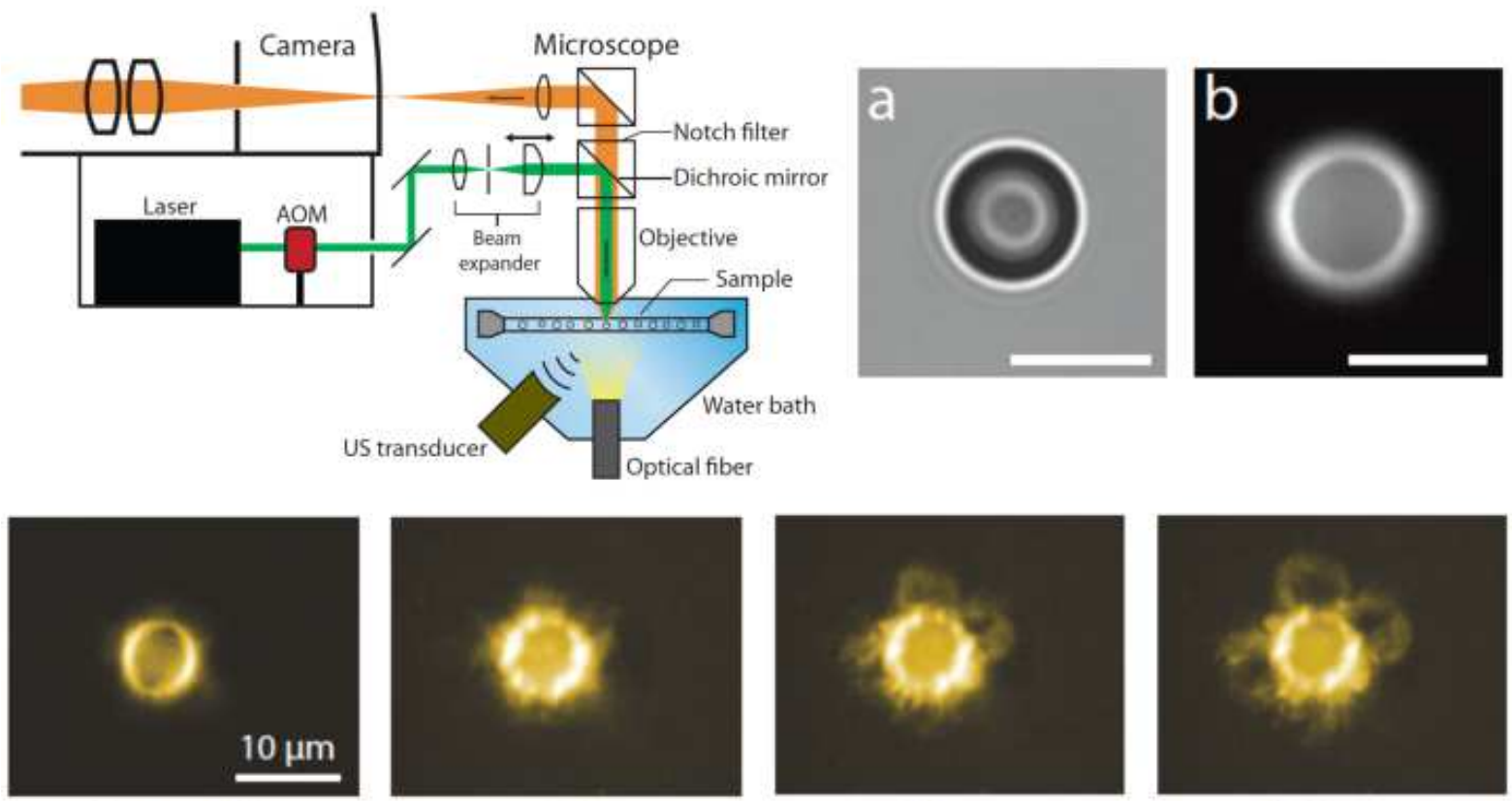

Before US

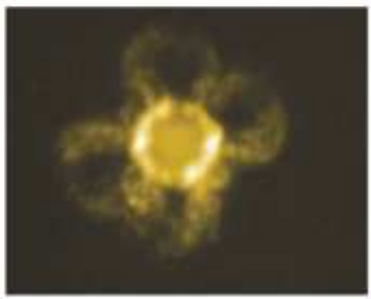

$\mathrm{t}=30 \mu \mathrm{s}$

$80 \mu \mathrm{s}$

$130 \mu \mathrm{s}$

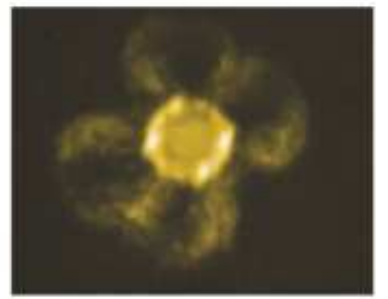

$330 \mu \mathrm{s}$

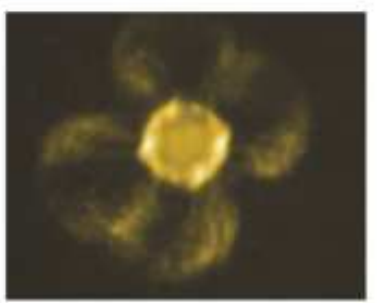

$430 \mu \mathrm{s}$

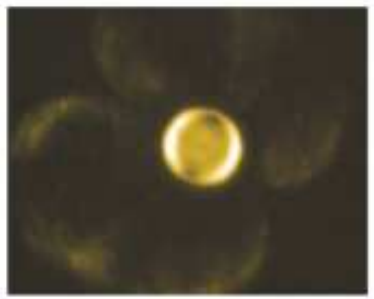

After US

Fig. 30 Schematic view of the experimental setup used for high-speed fluorescence recordings with the Brandaris-128. The laser light is focused by the imaging objective onto the sample. The beam-expander filters the laser beam and allows for adjustment of the laser spot size. Top right shows a bright-field image of a $4-\mu$ m microcapsule (a) and the very same capsule in fluorescence (b). The bottom section shows the release of liposomes (taken at 200 kfps) that were targeted to the phospholipid shell of the bubble. It displays strong acoustic streaming [21] and the potential for local drug release from bubbles driven by $\mathrm{MHz}$ frequency ultrasound (movie online).

applications. Fig. 30 shows a polymer-coated microcapsule (Philips Research) [128] imaged both in bright field and in fluorescence. The latter shows a distinct delineation of the wall boundaries and in addition a high contrast is achieved between the fluorescent liquid core and its surroundings. The microcapsules could be recorded with a frame rate of up to 10 million frames per second, giving a unique insight into the physical mechanisms of local intravascular drug delivery. Phospholipid-coated microbubbles loaded with liposomes are another important drugdelivery vehicle system [129] and the release of the drug-loaded liposomes is visualized in the lower section of Fig. 30. These images were recorded with a Photron SA 1.1 camera running at $200 \mathrm{kfps}$ with CW illumination controlled by the acousto-optic modulator, $\tau_{\text {laser }}=500 \mu \mathrm{s}$. The release is governed by the acoustical streaming pattern around the oscillating lipid microbubbles. 


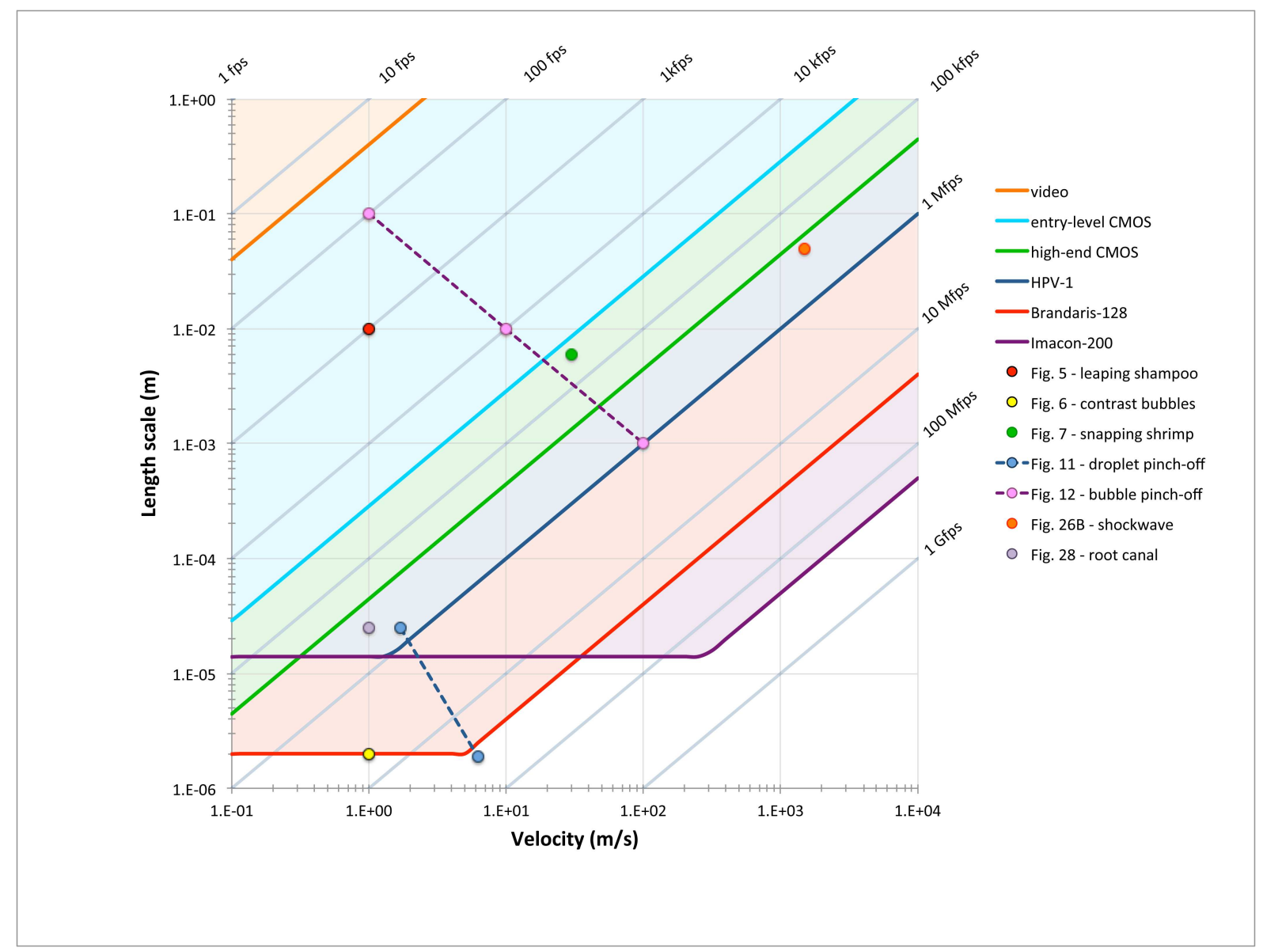

Fig. 31 High-speed imaging parameter chart. The typical velocity and typical length scale determine the frame rate. Several high-speed camera systems are included in this plot for reference. It then allows easy access to the optimum camera system. The dots refer to the examples given in this review.

\section{Summary}

All of the above has given us insight in the design of a high-speed imaging experiment. Let us now summarize all aspects in the following parameter chart, Fig. 31. The chart defines selection criteria for the experiment, based on Eqs. (1) and (2) and is supplemented with data from a series of high-speed imaging systems. This chart has been designed with the examples provided in this review, with the camera systems used in the examples. It can be easily adapted at will, for example to extend the parameter range, or to include custom camera systems.

The frame rates are represented by the diagonal lines and range from 1 frame per second at the top left to 1 billion frames per second at the bottom right. Any combination of the typical velocity and typical length scale of the problem gives the desired frame rate option. The selection of the camera is governed by two limiting cases, i.e. the minimum temporal resolution and the minimum spatial resolution. The minimum temporal resolution is dictated by the maximum frame rate, e.g. $25 \mathrm{fps}$ for video, 1 Mfps for the Shimadzu HPV-1, and 25 Mfps for the Brandaris-128 camera. Fig. 14 has demonstrated that the number of pixels is significantly reduced with 
increasing frame rate. We set the lowest imaging frame size to $100 \times 100$ pixels, which for an entry-level CMOS high-speed camera corresponds to a frame rate of $35 \mathrm{kfps}$. For a high-end CMOS high-speed camera the upper frame rate is then limited to $225 \mathrm{kfps}$. The minimum spatial resolution is given by the pixel size of the sensor and sets a limit to the minimum length scale that can be imaged in the experiment. We have set the minimum spatial sampling rate to $10 \times$ the Rayleigh criterion, in analogy to the temporal sampling rate in Eq. (1), and the maximum optical magnification was set to $100 \times$. For the Shimadzu HPV-1, for example, with its pixel size of approximately $70 \mu \mathrm{m}$, the lower limit was therefore set to $14 \mu \mathrm{m}$. The CMOS cameras, with a typical pixel size of $20 \mu \mathrm{m}$, have no issues with a minimum length scale within the parameter range set in the chart of Fig. 31. The two limiting cases for each camera system are indicated with the colored solid lines. The shaded areas indicate the selection of a specific camera system, assigned by the corresponding color. Examples presented in this review are indicated in the chart. For example, Figs. 5, 6, and 28 are displayed along the vertical line with velocity 1 $\mathrm{m} / \mathrm{s}$, while the other examples of Figs. 5, 7, and 26B are indicated along the horizontal line with a typical length scale of $1-5 \mathrm{~cm}$. It is seen that different camera systems are needed to image these events. This becomes even more evident for the two examples where scaling issues were present, Figs. 11 and 12. It can also be observed that several areas within the parameter space are exclusive to some specialized cameras. For example, micron-sized particles with a speed of $1 \mathrm{~m} / \mathrm{s}$ can only be imaged by a Brandaris-128 type camera, while sub-millimeter particles in water traveling at the speed of sound are to be imaged with a framing camera of the type Imacon-200. The chart also shows that micron-sized events at a speed of $1000 \mathrm{~m} / \mathrm{s}$ cannot be comfortably captured by the current range of high-speed imaging systems and until now we need to resort to single or double frames flash photography using iLIF, as was shown very recently for supersonic microjets by Tagawa et al. [130]. Emerging technologies in the near future will provide higher frame rates, and also better resolution and better sensitivity, which may, for example, lead to high-speed fluorescence imaging of flow phenomena at significantly increased frame rates. A better sensitivity in the end will be beneficial for the resolution anyway by a reduction of the pixel size. In the future we also hope for higher frame rates in more compact imaging systems.

The current possibilities of high-speed imaging systems are impressive, but new developments are imminent. New designs will in the very near future lead to increased frame rates, higher sensitivities, smaller pixel sizes, and an overall smaller form factor of the sensor. The improved technologies will allow us to work on unraveling new details in fine scale acoustics, such as high-frequency medical imaging and surface acoustic wave (SAW) atomization. It will be possible to study shockwave propagation at much smaller length scales. New application 
fields will emerge in plasma physics and eventually we may be able to follow light propagation in real-time, not only stroboscopically. With these kind of phenomena at a typical timescale of a nanosecond, this requires high-speed imaging at a frame rate of 1 billion frames per second. These systems may indeed become available in the near future, although we may encounter severe physical limitations in illumination and in the present IC semiconductor technology.

The purpose of this review was to provide insight in defining criteria for high-speed imaging experiments in fluids, to give rule-of-thumbs for a series of cases. To complete, let us therefore now also summarize three common pitfalls in high-speed imaging. The first pitfall is that one may be inclined to think that the velocity dictates the frame rate. How trivial it may sound but it is the relevant time scale that dictates the frame rate and the relevant time scale can be found from the ratio of the velocity and the length scale, see Sec. 2.2. The smaller the length scale the higher the frame rate, so for microfluidic applications this rule should definitely be particularly considered. Secondly, many researchers are tempted to use a high-speed camera no matter what, but in all honesty, high-speed cameras give lousy pictures because of their limited pixel count. If not ultimately necessary, it is advised to use still frames from high-speed photography, which give superior image resolution and significantly higher scientific value over the high-speed imaging frames. Agreed, we may need high-speed imaging to find all the details of the process under study, but in the end single flash or stroboscopic imaging will give more scientific satisfaction. And finally, please do not underestimate the illumination conditions for high-speed imaging. Many translate video recordings directly to high-speed recordings and assume that a factor 2 or maybe 10 is required to compensate for light loss, whereas in practice it amounts to an increase of three or four orders of magnitude simply dictated by the frame rate, viz. interframe time, let alone with an additional order of magnitude for setting a relevant exposure time.

Acknowledgements This hands-on review on high-speed imaging has not been possible without the numerous and valuable contributions of my dear colleagues and friends in the various fields of research. I would therefore like to acknowledge contributions and support from Detlef Lohse and Nico de Jong. I also enjoyed many scientific discussions with Claus-Dieter Ohl, Chao Sun, and Goji Etoh. I also gratefully acknowledge the valuable and extensive discussions with my former PhD students Erik Gelderblom, Arjan van der Bos, Aaldert Zijlstra, Bram Verhaagen, Christian Veldhuis and Wim van Hoeve. Many undergraduate students have contributed in the course of the Physics of Fluids lab course and Experimental Methods in Fluid Mechanics course and in particular I would like thank Tim Segers and Hans Kroes for contributions to Fig. 1C, Sander van der Meer and Ramon van den Berg for Fig. 3, Arthur van Bilsen and Raymond Bergmann for Fig. 8, and Rik Groenen, Elbert van Putten, Erik Gelderblom and Ramy El-Dardiry for contributions to Fig. 15. Furthermore, I would like 
to thank Devaraj van der Meer, Chien Ting 'Cash' Chin, Frits Mastik, Charles Lancée, Martijn Frijlink, Elmer Koene, Rik Vos, Manish Arora, Rory Dijkink, Marlies Overvelde, Jos de Jong, Valeria Garbin, Siggi Thoroddsen, Flordeliza Villanueva, Xucai Chen, Peter Andresen ${ }^{\dagger}$, Theo van der Meer, Marcus Aldén, Clemens Kaminski, Edwin van der Bunt, Jan Tukker, Mike Bailey, Larry Crum, and Tom Matula, and Hans Reinten and Mark van den Berg of Océ Technologies and Paul Duineveld of Philips for their contributions to this review. I would like to acknowledge various technical discussions with Frans Langeweg of Kodak MASD/Roper Scientific/Redlake, Vance Parker, Sid Nebeker and Nathan Nebeker of Cordin Company, Tim Nicholls of Photron, Kinko Tsuji and Yasushi Kondo of Shimadzu Corporation, and with John Boaler and Heiner Voges of LaVision. The technical skills of Henni Scholten ${ }^{\dagger}$, Gert-Wim Bruggert, Bas Benschop, Martin Bos, and of Jan Honkoop, Leo Bekkering, Wim van Alphen and Cees Pakvis of Erasmus MC are gratefully acknowledged.

\section{References}

1. D. Hu, B. Chan, J. Bush, Nature 424, 663 (2003)

2. S.N. Fry, R. Sayaman, M.H. Dickinson, Science 300, 495 (2003)

3. J. Glasheen, T. McMahon, Nature 380, 340 (1996)

4. M. Versluis, B. Schmitz, A. von der Heydt, D. Lohse, Science 289, 2114 (2000)

5. D. Lohse, B. Schmitz, M. Versluis, Nature 413, 477 (2001)

6. S.N. Patek, W.L. Korff, R.L. Caldwell, Nature 428, 819 (2004)

7. X.D. Shi, M.P. Brenner, S.R. Nagel, Science 265(5169), 219 (1994)

8. I. Cohen, M.P. Brenner, J. Eggers, S.R. Nagel, Phys. Rev. Lett. 83, 1147 (1999)

9. S.T. Thoroddsen, K. Takehara, Phys. Fluids 12, 1265 (2000)

10. S.T. Thoroddsen, J. Fluid Mech. 451, 371 (2002)

11. L. Xu, W. Zhang, S.R. Nagel, Phys. Rev. Lett. 94, 184505 (2005)

12. S.L. Anna, N. Bontoux, H.A. Stone, Appl. Phys. Lett. 82, 364 (2003)

13. W. van Hoeve, S. Gekle, J.H. Snoeijer, M. Versluis, M.P. Brenner, D. Lohse, Phys. Fluids 22(12), 122003 (2010)

14. A.U. Chen, O.A. Basaran, Phys. Fluids 14, L1 (2001)

15. B.J. de Gans, P.C. Duineveld, U.S. Schubert, Adv. Mater. 16(3), 203 (2004)

16. N. de Jong, P.J. Frinking, A. Bouakaz, M. Goorden, T. Schourmans, X. Jingping, F. Mastik, Ultrasound Med. Biol. 26, $487(2000)$

17. J.E. Chomas, P.A. Dayton, D. May, J. Allen, A. Klibanov, , K. Ferrara, Appl. Phys. Lett. 77(7), 1056 (2000)

18. A. Bouakaz, M. Versluis, N. de Jong, Ultrasound Med. Biol. 31(3), 391 (2005)

19. S.M. van der Meer, B. Dollet, C.T. Chin, A. Bouakaz, M. Voormolen, N. de Jong, M. Versluis, D. Lohse, J. Acoust. Soc. Am. 121(1), $648(2007)$

20. Y.A. Pishchalnikov, O.A. Sapozhnikov, M.R. Bailey, J. J. C. Williams, R.O. Cleveland, T. Colonius, L.A. Crum, A.P. Evan, J.A. McAteer, J Endourol. 17(7), 435 (2003)

21. P. Marmottant, S. Hilgenfeldt, Nature 423, 153 (2003)

22. C.D. Ohl, M. Arora, R. Ikink, N. de Jong, M. Versluis, M. Delius, D. Lohse, Biophys. J. 91, 4285 (2006) 
23. A.V. Wamel, K. Kooiman, M. Harteveld, M. Emmer, F.J. ten Cate, M. Versluis, N.D. Jong, J. Contr. Rel. 112(2), $149(2006)$

24. N. Kudo, K. Okada, K. Yamamoto, Biophys. J. 96(12), 4866 (2009)

25. H.E. Edgerton, J. J. R. Killian, Flash! Seeing the Unseen by Ultra High-Speed Photography (Boston C. T. Branford Co., Boston, 1954)

26. H.E. Edgerton, J. J. R. Killian, Flash! Seeing the Unseen by Ultra High-Speed Photography (Boston C. T. Branford Co., Boston, 1954), p. 41

27. H.E. Edgerton, E. Jussim, G. Kayafras, G. Hayafas, Stopping Time: The Photographs Of Harold Edgerton (Abrams, Harry N., Inc., 1987), p. 45

28. H.E. Edgerton, J. J. R. Killian, Flash! Seeing the Unseen by Ultra High-Speed Photography (Boston C. T. Branford Co., Boston, 1954), p. 130

29. A.M. Worthington, R.S. Cole, Phil. Trans. R. Soc., London, Ser. A 189, 137 (1897)

30. A.M. Worthington, R.S. Cole, Phil. Trans. R. Soc., London, Ser. A 194, 175 (1900)

31. W.H.F. Talbot, Phil. Mag. (4th series) January, 73 (1852)

32. C. Cranz, B. Glatzel, Phys. Gesell. 14, 525 (1912)

33. E. Mach, L. Mach, Sitzungsber. Kaiserl. Akad. Wissenschaft. Mathematisch-Naturwisenschaft. Classe 98, 1310 (1889)

34. A.M. Worthington, A study of splashes (Longmans, Green and Co., London, 1908)

35. S.T. Thoroddsen, T.G. Etoh, K. Takehara, J. Fluid Mech. 557, 63 (2006)

36. E. Muybridge, A.V. Mozley, Human and Animal Locomotion (Dover, New York, 1887)

37. E.J. Marey, C. R. Acad. Sci. 67, 1341 (1868)

38. E. Marey, Le mouvement (G. Masson, Paris, 1894)

39. B. Brixner, in 20th International Congress on High Speed Photography and Photonics, Proceedings, vol. 1801, ed. by J.M. Dewey, R.G. Racca (SPIE, Bellingham, WA, 1992), vol. 1801, pp. 52-60

40. C. Miller, Journal of the Society of Motion Picture Engineers 53, 479 (1949)

41. E.A. Igel, M. Kristiansen, Rotating-Mirror Streak and Framing Cameras, vol. PM43 (SPIE, Bellingham, WA, 1997)

42. V. Parker, C. Roberts, in High Speed Photography and Photonics, vol. PM120, ed. by S.F. Ray (SPIE, Bellingham, WA, 2002), pp. 167-180

43. J. Honour, in High Speed Photography and Photonics, vol. PM120, ed. by S.F. Ray (SPIE, Bellingham, WA, 2002), pp. $134-149$

44. A. Prosperetti, L.A. Crum, H.C. Pumphrey, Geophys. Res. 94, 3255 (1989)

45. C.T. Chin, C. Lancée, J. Borsboom, F. Mastik, M.E. Frijlink, N. de Jong, M. Versluis, D. Lohse, Rev. Sci. Instr. $\mathbf{7 4}(12), 5026(2003)$

46. J. Honour, in 21st International Congress on High Speed Photography and Photonics, Proceedings, vol. 2513 (SPIE, Bellingham, WA, 1994), vol. 2513, pp. 28-34

47. M. Minnaert, Phil. Mag. 16, 235 (1933)

48. M. Versluis, C. Blom, D. van der Meer, K. van der Weele, D. Lohse, J. Stat. Mech 280, P07007 (2006)

49. A. Kaye, Nature 197, 100 (1963) 
50. A.A. Collyer, P. Fisher, Nature 261, 682 (1976)

51. D. Lohse, R. Bergmann, R. Mikkelsen, C. Zeilstra, D. van der Meer, M. Versluis, J. van der Weele, M. van der Hoef, J. Kuipers, Phys. Rev. Lett. 93(19), 198003 (2004)

52. M. Versluis, N. Georgiev, L. Martinsson, M. Aldén, S. Kröll, Appl. Phys. B 65, 411 (1997)

53. M. Davidson, M. Abramowitz, in Encyclopedia of Imaging Science and Technology (2002), pp. 1106-1141

54. A. van der Bos, A. Zijlstra, E. Gelderblom, M. Versluis, Exp. Fluids 51, 12831289 (2011)

55. H. Dong, W.W. Carr, J.F. Morris, Rev. Sci. Instr. 77(8), 085101 (2006)

56. I.M. Hutchings, G.D. Martin, S.D. Hoath, J. Imaging Sci. Technology 51(5), 438 (2007)

57. J. de Jong, G. de Bruin, H. Reinten, M. van den Berg, H. Wijshoff, M. Versluis, D. Lohse, J. Acoust. Soc. Am. 120, $1257(2006)$

58. H. Wijshoff, Phys. Rep. 491(4-5), 77 (2010)

59. J.A.F. Plateau, Statique exprimentale et thorique des liquides soumis aux seules forces molculaires (Gauthier-Villard, Paris, 1873)

60. L. Rayleigh, Proc. R. Soc. London 29, 71 (1879)

61. S. Longuet-Higgins, B.R. Kerman, K. Lunde, J. Fluid Mech. 230, 365 (1991)

62. H.N. Oğuz, A. Prosperetti, J. Fluid Mech. 257, 111 (1993)

63. M. Gordillo, A. Sevilla, J. Rodríguez-Rodríguez, C. Martnez-Bazán, Phys. Rev. Lett. 95(19), 194501 (2005)

64. R. Bergmann, D. van der Meer, M. Stijnman, M. Sandtke, A. Prosperetti, D. Lohse, Phys. Rev. Lett. 96, 154505 (2006)

65. S.T. Thoroddsen, T.G. Etoh, K. Takehara, Phys. Fluids 19(4), 042101 (2007)

66. A.G. Zijlstra, C.D. Ohl, J. Acoust. Soc. Am. 123(1), 29 (2008)

67. T.G. Etoh, D. Poggemenn, A. Ruckelshausen, A. Theuwissen, G. Kreider, H.O. Folkerts, H. Mutoh, Y. Kondo, H. Maruno, K. Takubo, H. Soya, K. Takehara, T. Okinaka, Y. Takano, T. Reisinger, C. Lohmann, (2002), IEEE International Solid-State Circuits Conference Digest of Tech. Papers, pp. 46-47

68. P. Hoess, K. Fleder, in 24th International Congress on High-Speed Photography and Photonics, Proceedings, vol. 4183, ed. by K. Takayama, T. Saito, H. Kleine, E.V. Timofeev (SPIE, Bellingham, WA, 2003), vol. 4183, p. 127

69. F. Kun, F.K. Wittel, H.J. Herrmann, B.H. Kröplin, K.J. Måløy, Phys. Rev. Lett. 96, 025504 (2006)

70. J. Rensen, S. Luther, J. de Vries, D. Lohse, Int. J. Multiphase Flow 31, 285 (2005)

71. H. Bruun, Hot Wire Anemometry: Principles and Signal Analysis (Oxford Univesity Press, 1995)

72. E. Gelderblom, R. Vos, F. Mastik, T. Faez, T. Kokhuis, T. van der Steen, N. de Jong, D. Lohse, M. Versluis, Rev. Sci. Instr. (2012)

73. M. Versluis, P. Palanchon, D. Goertz, I. Heitman, S. van der Meer, B. Dollet, N. de Jong, D. Lohse, Phys. Rev. E 82, $026321(2010)$

74. R.J. Adrian, J. Westerweel, Particle Image Velocimetry (Cambridge University Press, 2011)

75. T.G. Etoh, D.H. Nguyen, S.V.T. Dao, C.L. Vo, M. Tanaka, K. Takehara, T. Okinaka, H.V. Kuijk, J. Bosiers, M. Lesser, D. Ouellete, M. Maruyama, T. Hayashida, T. Arai, (2011), IEEE International Solid-State Circuits Conference Digest of Tech. Papers, pp. 406-407 
76. Y. Tichigi, K. Hanzawa, Y. Kato, R. Kuroda, H. Mutoh, R. Hirose, H. Tominaga, K. Takubo, Y. Kondo, S. Sugawa, (2012), IEEE International Solid-State Circuits Conference Digest of Tech. Papers, pp. 382-384

77. G.S. Settles, Schlieren and Shadowgraph Techniques (Springer-Verlag, Berlin, Heidelberg, New York, 2001)

78. A.W.G. de Vries, A. Biesheuvel, L. van Wijngaarden, Int. J. Multiphase Flow 28, 1823 (2002)

79. C. Brückner, Phys. Fluids 11, 1781 (1999)

80. L. Schouveiler, M. Provansal, Phys. Fluids 14, 3846 (2002)

81. C. Veldhuis, A. Biesheuvel, L. van Wijngaarden, D. Lohse, Nonlinearity 18(1), C1 (2005)

82. L. van Wijngaarden, J. Fluid Mech. 541, 203 (2005)

83. M. Bailey, V. Khokhlova, O. Sapozhnikov, S. Kargl, L. Crum, Acoustical Physics 49, 369 (2003)

84. O.A. Sapozhnikov, A.D. Maxwell, B. MacConaghy, M.R. Bailey, J. Acoust. Soc. Am. 121(2), 1190 (2007)

85. S.J. Warden, Sports Medicine 33, 95 (2003)

86. X. Zhen, A. Ludomirsky, L.Y. Eun, T.L. Hall, B.C. Tran, J.B. Fowlkes, C.A. Cain, IEEE Trans. Ultrason. Ferroelec. Freq. Contr. 51(6), $726(2004)$

87. J.E. Kennedy, G.R. ter Haar, D. Cranston, Br. J. Radiol 76(909), 590 (2003)

88. N. Bremond, M. Arora, C. Ohl, D. Lohse, Phys. Rev. Lett. 96, 224501 (2006)

89. C.D. Ohl, Phys. Fluids 14(10), 3512 (2002)

90. T. Dreyer, W. Krauss, E. Bauer, R.E. Riedlinger, (2000), IEEE Ultrasonics Symposium, Proceedings, vol. 2, pp. $1239-1242$

91. W. Merzkirch, Flow Visualization, $2^{\text {nd }}$ ed. (Academic Press, Inc., New York, 1987)

92. H. Kleine, Eur. Phys. J. Special Topics 182, 3 (2010)

93. J. Kitzhofer, T. Nonn, C. Brückner, Exp. Fluids 51, 1471 (2011)

94. M.P. Arroyo, K.D. Hinsch, Recent developments of PIV towards 3D measurements (Springer-Verlag, Berlin, Heidelberg, 2008), Topics in Appl. Physics, vol. 112, pp. 127-154

95. C.D. Ohl, T. Kurz, R. Geisler, O. Lindau, W. Lauterborn, Phil. Trans. R. Soc. A 357, 269 (1999)

96. H. Kleine, H. Olivier, K. Tsuji, K. Etoh, K. Takehara, T.G. Etoh, Time-Resolved Mach-Zehnder Interferometry of Shock Waves (Springer-Verlag, Berlin, Heidelberg, 2012), 28th International Symposium on Shock Waves, vol. 1, pp. $577-583$

97. J.M. Desse, Opt. Laser Eng. 44, 304 (2006)

98. R.C.A. van der Veen, T. Tran, D. Lohse, C. Sun, Phys. Rev. E 85, 026315 (2012)

99. A.L. Porta, G.A. Voth, A.M. Crawford, J. Alexander, E. Bodenschatz, Nature 409, 1017 (2001)

100. N. Mordant, E. Leveque, J. Pinton, New J. Phys. 6, 34 (2004)

101. F. Toschi, E. Bodenschatz, Ann. Rev. Fluid Mech. 41(1), 375 (2009)

102. Y. Gasteuil, W.L. Shew, M. Gibert, F. Chillá, B. Castaing, J.F. Pinton, Phys. Rev. Lett. 99, 234302 (2007)

103. R. Ni, S.D. Huang, K.Q. Xia, J. Fluid Mech. 692, 395 (2012)

104. J. Rensen, S. Luther, D. Lohse, J. Fluid Mech. 538, 153 (2005)

105. J. Martínez-Mercado, D. Chehata, D. van Gils, C. Sun, D. Lohse, J. Fluid Mech. 650, 287 (2010)

106. J. Martínez-Mercado, V.N. Prakash, Y. Tagawa, C. Sun, D. Lohse, Phys. Fluids 24, 055106 (2012) 
107. K. Hoyer, M. Holzner, B. Luethi, M. Guala, A. Liberzon, W. Kinzelbach, Exp. Fluids 39, 923 (2005)

108. J. Tilton, Fluid and particle dynamics, Perrys Chemical Engineers Handbook, vol. 6.150 (1999)

109. M. Raffel, C. Willert, J. Kompenhans, Particle Image Velocimetry, 2nd edn. (Springer, Berlin, Germany, 1998)

110. C. Boutsioukis, B. Verhaagen, M. Versluis, E. Kastrinakis, P.R. Wesselink, L.W.M. van der Sluis, J. Endod. 36(5), $875(2010)$

111. C. Meinhart, S. Wereley, M. Gray, Measurement Science and Technology 11, 809 (2000)

112. B. Verhaagen, C. Boutsioukis, G.L. Heijnen, L.W.M. van der Sluis, M. Versluis, Exp. Fluids (2012)

113. M.S. Pommer, A.R. Kiehl, G. Soni, N.S. Dakessian, C.D. Meinhart, (2007), Proceedings of the 2nd IEEE International Conference on Nano/Micro Engineered and Molecular Systems

114. C. Poelma, J.M. Mari, N. Foin, M.X. Tang, R. Krams, C.G. Caro, P.D. Weinberg, J. Westerweel, Exp. Fluids 50, 777 $(2011)$

115. T. Ding, T.H. van der Meer, M. Versluis, M. Golombok, J. Hult, M. Aldén, C.F. Kaminski., (2000), Third International Symposium on Turbulence, Heat and Mass Transfer, Proceedings, pp. 857-864

116. P.A. Nooren, M. Versluis, T.H. van der Meer, R.S. Barlow, J.H. Frank, Appl. Phys. B 71, 95 (2000)

117. W.J.A. Dahm, P.E. Dimotakis, AAIA 25(9), 1216 (1987)

118. K.R. Sreenivasan, R.R. Prasad, Physica D 38(1-3), 322 (1989)

119. J.P. Crimaldi, Exp. Fluids 44, 851 (2008)

120. A.C. Eckbreth, Laser Diagnostics for Combustion Temperature and Species, 2nd edn. (Gordon and Breach, UK, 1996)

121. K. Kohse-Höinghaus, J.B. Jeffries, Applied Combustion Diagnostics (Taylor and Francis, New York, 2002)

122. M. Aldén, J. Bood, Z. Li, M. Richter, Proc. of the Comb. Inst. 33, 69 (2011)

123. C. Poelma, P. Vennemann, R. Lindken, J. Westerweel, Exp. Fluids 45(4), 703 (2008)

124. B. Hiller, R.A. Booman, C. Hassa, R.K. Hanson, Rev. Sci. Instr. 55(12), 1964 (1984)

125. C.P. Gendrich, M.M. Koochesfahani, D.G. Nocera, Exp. Fluids 23(5), 361 (1997)

126. R.B. Miles, J. Grinstead, R.H. Kohl, G. Diskin, Measurement Science and Technology 11(9), 1272 (2000)

127. L.A. Ribarov, J.A. Wehrmeyer, R.W. Pitz, R.A. Yetter, Appl. Phys. B 74(2), 175 (2002)

128. K. Kooiman, M.R. Böhmer, M. Emmer, H.J. Vos, C. Chlon, W.T. Shi, C.S. Hall, S.H.P.M. de Winter, K. Schroën, M. Versluis, N. de Jong, A. van Wamel, J. Contr. Rel. 133, 109 (2009)

129. I. Lentacker, S.C. De Smedt, N.N. Sanders, Soft Matter 5(11), 2161 (2009)

130. Y. Tagawa, N. Oudalov, C.W. Visser, I. Peters, D. van der Meer, C. Sun, A. Prosperetti, D. Lohse, Phys. Rev. X 2, 031002 (2012) 